\title{
Tandem Cylinder Noise Predictions
}

\author{
David P. Lockard, Mehdi R. Khorrami†, Meelan M. Choudhari†, \\ Florence V. Hutcheson; Thomas F. Brooks ${ }^{\S}$ \\ NASA Langley Research Center, Hampton, VA 23681 \\ and \\ Daniel J. Stead ${ }^{\top}$ \\ Lockheed Martin Engineering and Sciences, Hampton, VA 23681
}

\begin{abstract}
In an effort to better understand landing-gear noise sources, we have been examining a simplified configuration that still maintains some of the salient features of landing-gear flow fields. In particular, tandem cylinders have been studied because they model a variety of component level interactions. The present effort is directed at the case of two identical cylinders spatially separated in the streamwise direction by 3.7 diameters.

Experimental measurements from the Basic Aerodynamic Research Tunnel (BART) and Quiet Flow Facility (QFF) at NASA Langley Research Center (LaRC) have provided steady surface pressures, detailed off-surface measurements of the flow field using Particle Image Velocimetry (PIV), hot-wire measurements in the wake of the rear cylinder, unsteady surface pressure data, and the radiated noise. The experiments were conducted at a Reynolds number of $166 \times 10^{5}$ based on the cylinder diameter. A trip was used on the upstream cylinder to insure a fully turbulent shedding process and simulate the effects of a high Reynolds number flow.

The parallel computational effort uses the three-dimensional Navier-Stokes solver CFL3D with a hybrid, zonal turbulence model that turns off the turbulence production term everywhere except in a narrow ring surrounding solid surfaces. The current calculations further explore the influence of the grid resolution and spanwise extent on the flow and associated radiated noise. Extensive comparisons with the experimental data are used to assess the ability of the computations to simulate the details of the flow. The results show that the pressure fluctuations on the upstream cylinder, caused by vortex shedding, are smaller than those generated on the downstream cylinder by wake interaction. Consequently, the downstream cylinder dominates the noise radiation, producing an overall directivity pattern that is similar to that of an isolated cylinder. Only calculations based on the full length of the model span were able to capture the complete decay in the spanwise correlation, thereby producing reasonable noise radiation levels.
\end{abstract}

\section{Nomenclature}

$\begin{array}{ll}b & \text { span } \\ D & \text { cylinder diameter } \\ F x y z & \text { fine grid } \\ k & \text { wavenumber }=\frac{2 \pi}{\lambda} \\ M & \text { Mach number } \\ M x y z & \text { medium grid } \\ M x y F z & \text { medium grid in } \mathrm{x}-\mathrm{y} \text { plane, fine in the span } \\ p & \text { pressure } \\ u, v, w & \text { Cartesian fluid velocity components } \\ U, V, W & \text { time-averaged velocity components } \\ x, y, z & \text { Cartesian coordinates }\end{array}$
Greek:
$\lambda \quad$ acoustic wavelength
$\rho \quad$ fluid density
$\theta \quad$ azimuthal angle

Superscript:

, perturbation quantity (e.g. $\rho^{\prime}=\rho-\rho_{\infty}$ )

Subscript:

$\infty \quad$ freestream quantity

*Aerospace Technologist, Computational AeroSciences Branch, Mail Stop 128, Senior Member, AIAA

${ }^{\dagger}$ Aerospace Technologist, Computational AeroSciences Branch, Mail Stop 128, Associate Fellow, AIAA

$¥$ Aerospace Technologist, Aeroacoustics Branch, Mail Stop 461, Member, AIAA

$\S$ Aerospace Technologist, Aeroacoustics Branch, Mail Stop 461, Fellow, AIAA

ब Aerospace Engineer, Aeroacoustics Branch, Mail Stop 461

This material is declared a work of the U.S. Government and is not subject to copyright protection in the United States.2007 


\section{Introduction}

In an effort to better understand landing gear noise sources, we have been examining a simplified configuration that still maintains some of the salient features of landing gear flow fields. In particular, tandem cylinders have been studied because they model a variety of component level interactions. The present effort is directed at the case of two identical cylinders spatially separated in the streamwise direction. Extensive experimental data have been collected to investigate the flow fields, but also to provide benchmark data to assess the ability of computational simulations to capture the relevant features of the flow. Khorrami et al. ${ }^{1}$ have already presented some comparisons with the relevant results in the literature.

The Basic Aerodynamic Research Tunnel (BART) and Quiet Flow Facility (QFF) at NASA Langley Research Center (LaRC) have been used to gather the experimental data. ${ }^{2-4}$ The parallel computational effort by Khorrami et al. ${ }^{5,6}$ initially was restricted to two-dimensional (2-D), fully turbulent simulations using standard, unsteady Reynolds averaged Navier-Stokes (URANS) equations in conjunction with Menter's ${ }^{7}$ two-equation, Shear Stress Transport (SST) turbulence model. Separation distances of $\mathrm{L} / \mathrm{D}=1.435$ and 3.7 were considered, where $\mathrm{D}$ is the diameter and $\mathrm{L}$ the distance between the cylinder centroids. In the case of short separations, $\mathrm{L} / \mathrm{D}<2.4$, the two cylinders behave as a single bluff body with vortex shedding only occurring at the rear cylinder. ${ }^{8}$ For large separation distances, such as $\mathrm{L} / \mathrm{D}=3.7$, the flow field on the upstream cylinder approaches that of an isolated single cylinder, and both cylinders exhibit shedding.

The 2-D computational results showed reasonable overall agreement with the measured data but failed to match the details, especially those of the off-surface mean and fluctuating velocity fields. Apart from the shortcomings associated with the 2-D nature of the simulations, those computations produced very strong, coherent shedding without any small scale structures, presumably because of the overly diffusive nature of the turbulence model. Considerable improvement in the comparisons with experiment was obtained by Khorrami et al. ${ }^{1}$ by extending the computations to three-dimensions and using a hybrid, zonal turbulence model that turned off the turbulence production term everywhere except in a narrow ring surrounding the solid surfaces. Due to the high computational cost of the 3D simulations, the spanwise extent of the computational domain was restricted to 3 cylinder diameters. Computations with the hybrid turbulence model more faithfully reproduced the three dimensionality in the flow, in contrast to 3-D URANS calculations that had shown almost no three-dimensional behavior. Limited calculations with an extended spanwise domain of 6 cylinder diameters demonstrated the influence of the span on the lateral correlation length.

An extensive review of the literature on cylinder flows can be found in Zdravkovich, ${ }^{9,10}$ and work of particular relevance to the current study on tandem cylinders is given in references 8 and 11 . Khorrami et al. ${ }^{1}$ have already given a detailed summary of our previous work, and examined the details of the tandem cylinder flow field based on work in the literature.

In this paper, we focus our attention on a streamwise separation of the cylinders of 3.7 diameters. We extend our previous work by presenting initial flow and aeroacoustic measurements from the QFF. The purpose of the QFF experiment is twofold. First, we reproduce some of the previous findings from the BART to evaluate facility independence. Secondly, the QFF is an anechoic wind tunnel which allows for acoustic measurements. Some microphone data giving the radiated sound field is used in the comparisons with the computational results. The simulations further investigate the influence of the grid resolution and spanwise extent on the solutions.

\section{Experiments}

The simulated tandem cylinder configuration is comprised of two cylinders of equal diameter (D) aligned along the streamwise direction. The geometry under consideration is shown in figure 1 . The separation distance, $\mathrm{L}$, between the cylinders is $3.7 \mathrm{D}$. The diameter of the cylinders is 2.25 inches $(0.05715 \mathrm{~m})$. A separation distance of $1.435 \mathrm{D}$ has also been examined, but will not be addressed in this paper. The angle $\theta$ is measured from the upstream stagnation point and is positive in the clockwise direction.

Two experiments by Jenkins et al..$^{2,3}$ have been performed in the BART, providing steady surface pressures, detailed off-surface measurements of the flow field using Particle Image Velocimetry (PIV), hot-wire measurements in the wake of the downstream cylinder, and unsteady surface pressure data. The BART is a subsonic, atmospheric wind tunnel used to investigate the fundamental characteristics of complex flow fields (see figure 2(a)). The tunnel has a closed test section with a height of 28 inches $(0.711 \mathrm{~m})$, a width of 40 inches $(1.016 \mathrm{~m})$, and a length of 120 inches $(3.048 \mathrm{~m})$. The free stream velocity was set to $144 \mathrm{ft} / \mathrm{s}(44 \mathrm{~m} / \mathrm{s})$ to achieve a Reynolds number based on cylinder diameter of $1.66 \times 10^{5}$. At these conditions the free stream turbulence level was less than $0.10 \%$. The cylinders spanned the entire BART tunnel height, such that the aspect ratio during the experiment was $\mathrm{b} / \mathrm{D}=12.4$. To ensure a fully turbulent shedding process, the boundary layers on the upstream cylinder were tripped between azimuthal locations of 50 and 60 degrees from the leading stagnation point. The measured surface pressure distribution for the large separation case was nearly identical 
to that measured by previous investigators for a single, isolated cylinder at a Reynolds number greater than 8 million. ${ }^{12}$ Additional information about the BART experiments can be found in References 2 and 3.

Previous QFF measurements documenting the fluctuations in the wake and the radiated sound field for various isolated cylinder arrangements have been performed by Hutcheson and Brooks. ${ }^{4}$ The identical tandem cylinder arrangements tested in the BART have now been investigated in the QFF. The QFF is an open jet facility specifically designed for anechoic testing and is equipped with a $2 \mathrm{ft}(0.61 \mathrm{~m}) \times 3 \mathrm{ft}(0.914 \mathrm{~m})$ rectangular open jet nozzle. The test models were supported above the nozzle by two vertical side plates that were mounted to the short sides of the nozzle (see figure 2(b)). The cylinders spanned the 3 $\mathrm{ft}(0.914 \mathrm{~m})$ section yielding an aspect ratio of $\mathrm{b} / \mathrm{D}=16$. To simulate the BART results as closely as possible, the trip arrangement on the front cylinder was duplicated, and
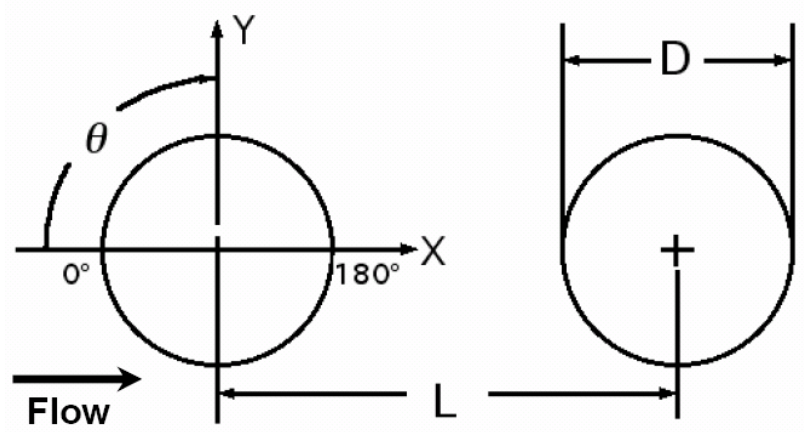

Figure 1. Schematic of tandem cylinder configuration. the speed in the tunnel was adjusted until the shedding frequency was matched. The nominal Mach number for the test was $0.1274(43.4 \mathrm{~m} / \mathrm{s})$ with a dynamic pressure of $0.166 \mathrm{psi}(1145 \mathrm{~Pa})$.

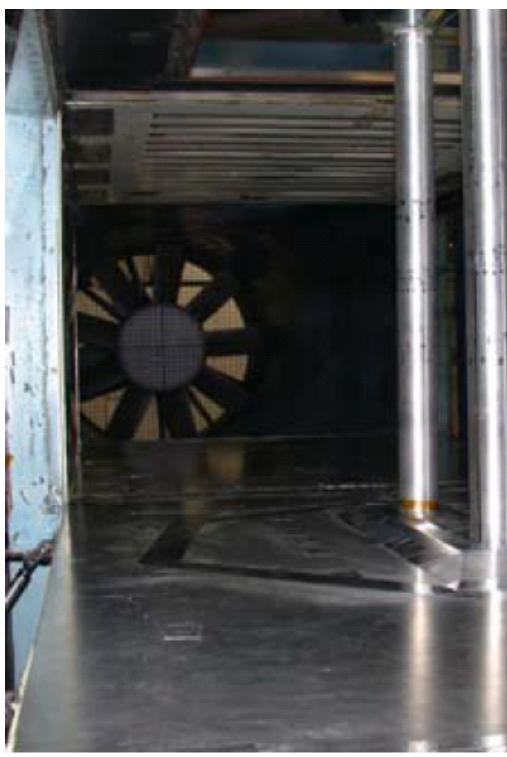

(a) BART
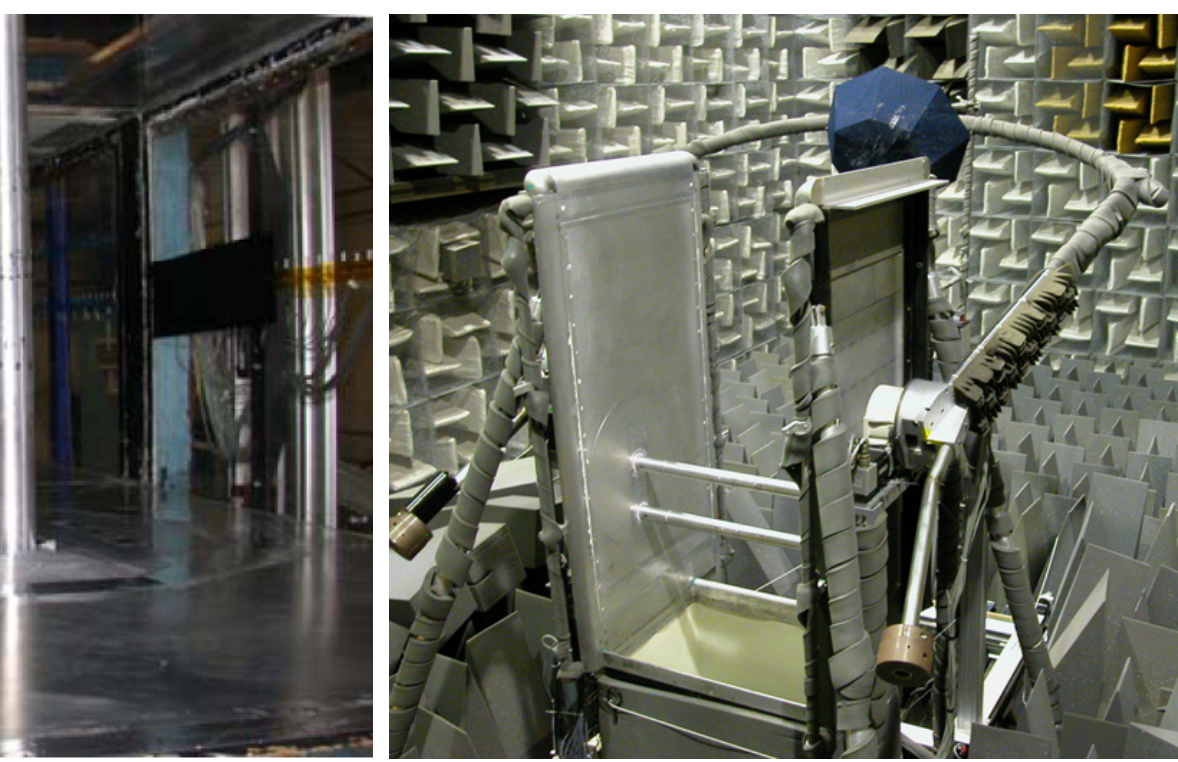

(b) QFF

Figure 2. Tandem cylinder arrangement in the Basic Aerodynamic Research Tunnel and Quiet Flow Facility.

Static and unsteady surface pressure measurements taken in the BART were duplicated in the QFF yielding valuable information about the facility dependence of the results. In addition, figure 3(a) shows the locations of three 1/8" microphones mounted on poles near the center of the span of the model. These microphones were located outside of the jet flow and were used to collect acoustic data. Microphone A was slightly upstream of the model at (-8.33D, $27.815 \mathrm{D})$ relative to the center of the upstream cylinder. Microphone B was slightly downstream at $(9.11 \mathrm{D}, 32.49 \mathrm{D})$, and microphone $\mathrm{C}$ was further downstream at $(26.55 \mathrm{D}, 27.815 \mathrm{D})$. These microphones were not equidistant from the model, but from the point $(9.11 \mathrm{D},-2.4 \mathrm{D})$. Points $\mathrm{A}$ and $\mathrm{B}$ were nearly equidistant from the center of the rear cylinder, and the $\mathrm{dB}$ correction to account for the distance to point $\mathrm{C}$ is $-1.5 \mathrm{~dB}$. The cylinder's primary shedding frequency of $178 \mathrm{~Hz}$ was so low that these pole mounted microphones were only about one wavelength from the model. Figure 3(b) shows the spectra from the three microphones. No shear layer corrections have been applied to this data. For the low frequency of the primary tone, the corrections are unlikely to have a noticeable effect. The peak amplitude was highest for the center microphone $\mathrm{B}$, but the upstream microphone $\mathrm{A}$ has the highest broadband levels and has more distinct peaks at both the second and third harmonics. Harmonic peaks have also been commonly observed for isolated cylinders. ${ }^{13}$

Unsteady surface pressure data were obtained from Endevco transducers. The data from both a spanwise row and a $45^{\circ}$ azimuthally spaced ring of sensors were obtained simultaneously with the microphone channels. The unequally spaced spanwise row was typically placed at $135^{\circ}$, but the cylinders were rotated in $15^{\circ}$ increments to obtain data at 


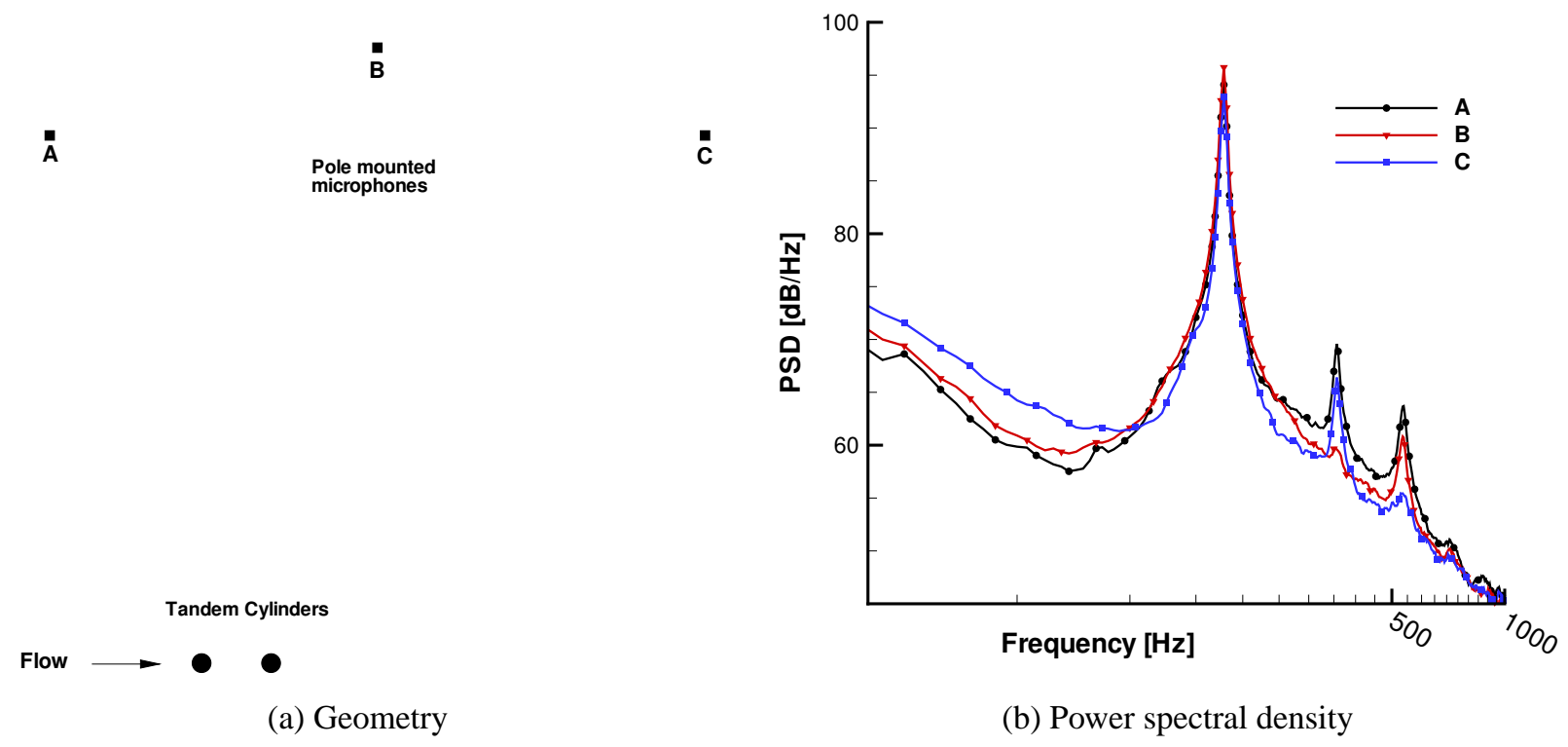

Figure 3. Schematic of microphone locations and spectra.

other angles. The rotations also provided more refined information about the azimuthal variation of the unsteady pressure from the centerline ring. Only the baseline $135^{\circ}$ configuration has been tested in the BART.

A 64-channel, 16 bit transient data recorder controlled by a workstation was used to acquire the data at a sampling rate of $25.6 \mathrm{kHz}$. High pass and low pass filters, set respectively at $5 \mathrm{~Hz}$ and $10 \mathrm{kHz}$, were used to condition the outputs from each microphone and Endevco channel. The noise spectra were obtained by partitioning each time signal into 1000 non-overlapping segments of 8192 samples, and each time history segment was Fourier transformed using a Hamming window for signal conditioning. The resulting frequency resolution was $3.125 \mathrm{~Hz}$.

The experimental results for the surface pressure coefficient from the two tunnels are compared in figure 4(a). Despite the differences between the open-jet and closed-wall tunnels, the agreement is quite good. The root-mean-square (rms) fluctuation levels obtained from the tests are compared in figure 4(b). For QFF data points with sufficient repeat runs, bars are included in the plot representing the scatter between different runs. However, they do not indicate a formal error band. The dotted lines in the plots are spline curve fits showing the general trends. The QFF $C p_{r m s}^{\prime}$ levels are slightly lower on the upstream cylinder, but the difference is somewhat more pronounced on the downstream cylinder. The higher resolution azimuthal data in the QFF results clearly shows the secondary peak in the rms levels near $120^{\circ}$ on the downstream cylinder. Oil flow patterns from the BART test ${ }^{3}$ indicated separation on the rear cylinder around $130^{\circ}$.

The correlation from the spanwise row of transducers is compared in figure 5. For spanwise separations greater than one diameter, the $\mathrm{BART}^{3}$ results are slightly higher than for the QFF, but are within the scatter in the data. There really is no appreciable difference between the correlation on the upstream and downstream cylinders. According to the data from Fujita et al. ${ }^{14}$ the correlation should drop rapidly and be highly scattered at high Reynolds numbers. Our result resembles those at high subcritical Reynolds numbers as shown by Szepessy. ${ }^{15}$ Apparently, the trip has a pronounced effect on the spanwise correlation. According to Zdravkovich, ${ }^{9}$ surface roughness enhances coherent shedding and tends to improve the spanwise uniformity by fixing the separation point. Perhaps the trip is doing something similar.

Instead of using the correlation, comparisons with simulations will primarily focus on the coherence at the shedding frequency. Figure 6 compares the correlation and coherence results from the QFF data. The coherence allows one to examine individual frequencies, and there is much less scatter in the data. All of the QFF data have been processed with the bin width of $3.125 \mathrm{~Hz}$. To facilitate the comparisons with the simulation data where much larger bins are needed because a much shorter time record is available, the experimental coherence was calculated by using an energy weighted average of the coherence over 7 bins (the bin containing $178 \mathrm{~Hz}$ and three on each side).

\section{Computational Simulations}

This study incorporates a coordinated effort between computations and experiment. The time-dependent CFD simu- 


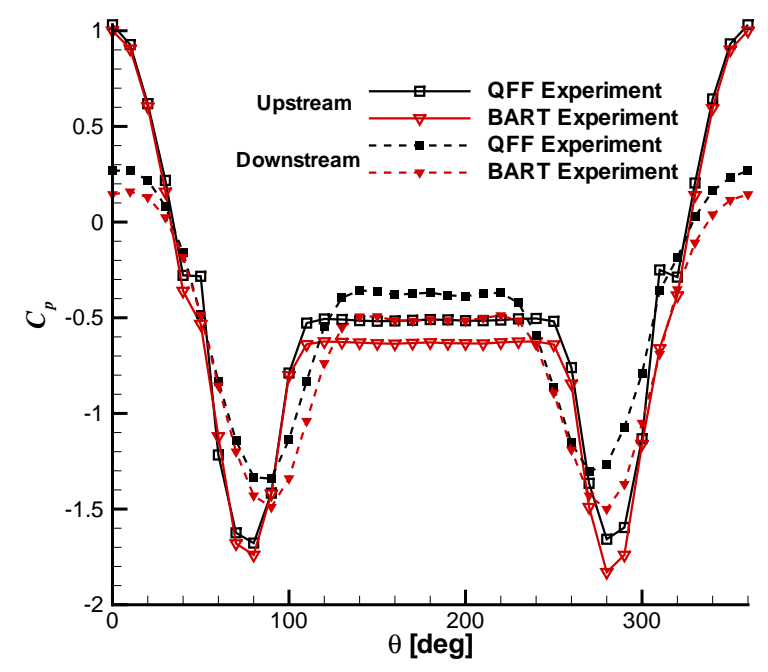

(a) $C p$

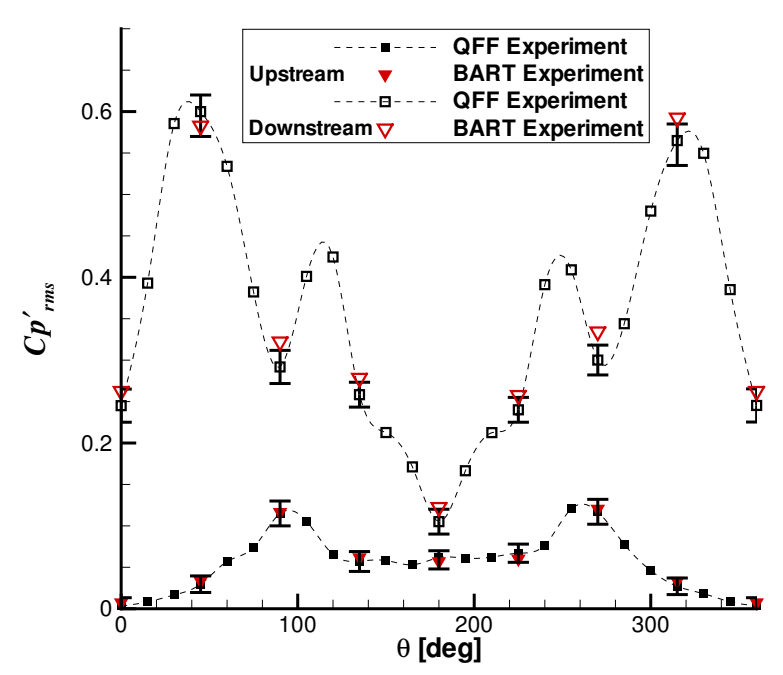

(b) $C p_{r m s}^{\prime}$

Figure 4. Comparison of $C p$ and $C p_{r m s}^{\prime}$ results from the BART ${ }^{3}$ and QFF experiments for the upstream and downstream cylinders.

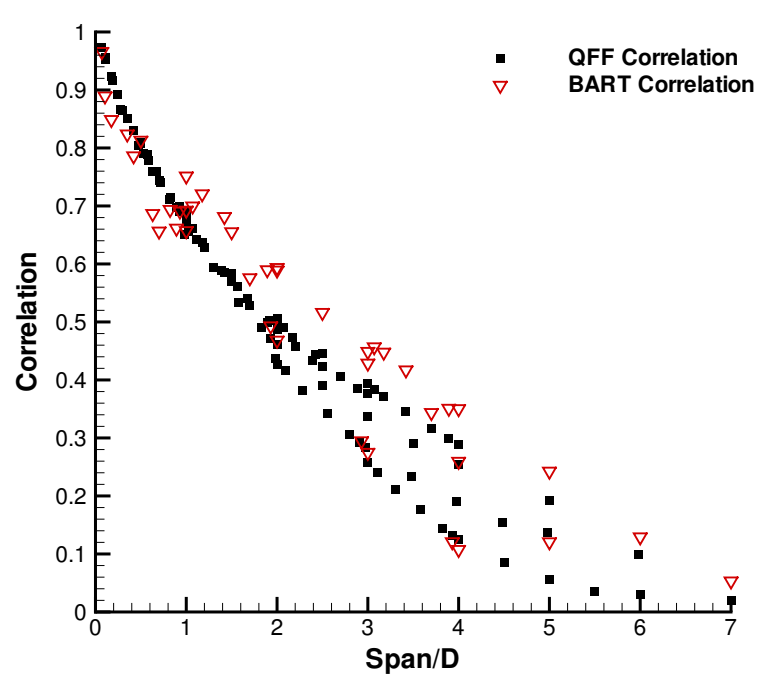

(a) Upstream cylinder

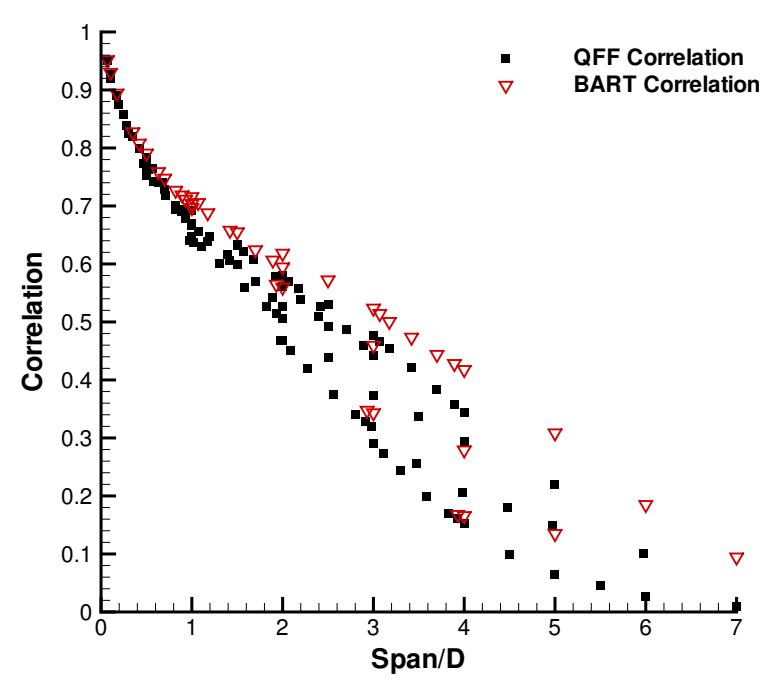

(b) Downstream cylinder

Figure 5. (a) Comparison of correlation results at $135^{\circ}$ from the BART ${ }^{3}$ and QFF experiments. 


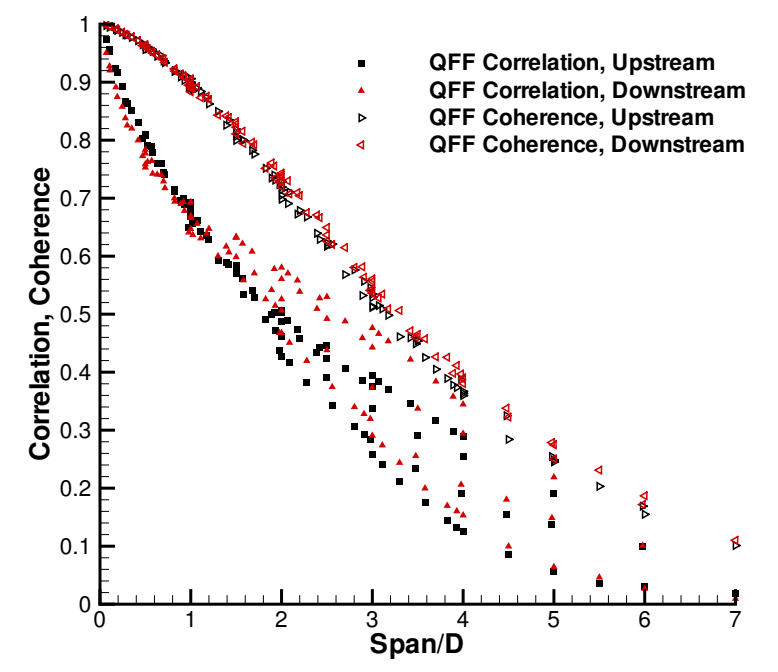

Figure 6. Comparison of the correlation and coherence at the shedding frequency from the QFF experiment.

lations have been performed using the second-order code CFL3D. ${ }^{16,17}$ CFL3D was developed at NASA LaRC to solve the 3-D, time-dependent, thin layer Reynolds-averaged Navier-Stokes (RANS) equations using a finite-volume formulation. The unsteady calculations use the shear stress transport (SST) $k-\omega$ turbulence model of Menter, ${ }^{7,18}$ which was developed for steady flow. Due to the overly diffusive nature of the turbulence model, results from three-dimensional URANS simulations remained essentially two-dimensional. To remedy this shortcoming, the quasi-laminar approach described in Khorrami et al. ${ }^{1}$ has been adopted for all three-dimensional computations. Following this approach, the flow field was assumed to be quasi-laminar, except for a narrow strip surrounding the cylinder surfaces where the SST turbulence model was used. Outside of this strip, the production term associated with the turbulence model was switched off. For computational convenience, the distance from the cylinder at which the switch occurs was set based on a set number of grid points from the surface. Because of the azimuthal nonuniformity in the grid stretching, the switch occurs between 0.01D and 0.04D, which includes the entire boundary layer within the region of attached flow. For different grid spacings, the number of points from the surface was changed to maintain the switching at the same physical distance.

The free stream Mach number in the computations was 0.166 , and the Reynolds number was $1.66 \times 10^{5}$ based on the cylinder diameter. The experiments were run at slightly different speeds, so the data have to be corrected to an equivalent speed. The QFF Mach number of 0.1274 was used as the baseline. Although the experiments used a trip strip to insure turbulent shedding, directly computing the effects of the trip is infeasible. Instead, the computations were run in a fully turbulent mode and allowed to transition on their own. The separation point on the upstream cylinder is critical in setting up the correct flow field. In the experiments, the separation was between $90^{\circ}-100^{\circ}$, and both cylinders shed. If separation occurs earlier than $90^{\circ}$, the wake of the upstream cylinder can reattach on the rear cylinder making them shed as one bluff body. Hence, the boundary layer produced by the turbulence model is essential to obtain reasonable results. In our calculations, the transition occurs early enough that the flow remains attached to the correct angle for turbulent flow. Even on the rear cylinder, the turbulence model is needed to obtain proper boundary layer development and separation. Although the interaction of the wake of the upstream cylinder on the downstream cylinder is the primary cause of the fluctuations, the interaction does not dictate the separation on the rear cylinder as our calculations with the turbulence model production turned off around the rear cylinder have borne out. Results are better around the rear cylinder with the turbulence model active in its boundary layer, but there is a need for improved models as resolving all the relevant phenomena is impractical.

\section{III.A. Configurations and Grids}

The tandem cylinders were simulated in free air and do not include any of the wind tunnel walls. The block structured, $\mathrm{x}-\mathrm{y}$ planar grid shown in figure 7 contained a total of 404,000 points in 92 blocks. Care was taken so that the first point off the solid surfaces was at $y^{+}<1$. To generate the 3-D grids, the planar grids were replicated along the span. The reference length in the grid was the cylinder diameter, D. Hence, a span of 3 means 3 diameters. Periodic boundary 


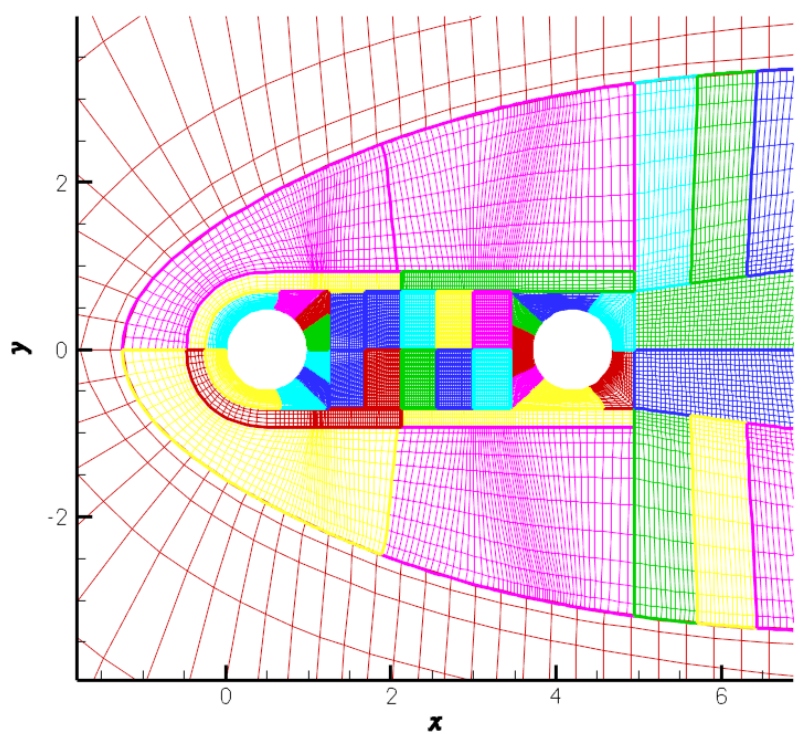

(a) Near field

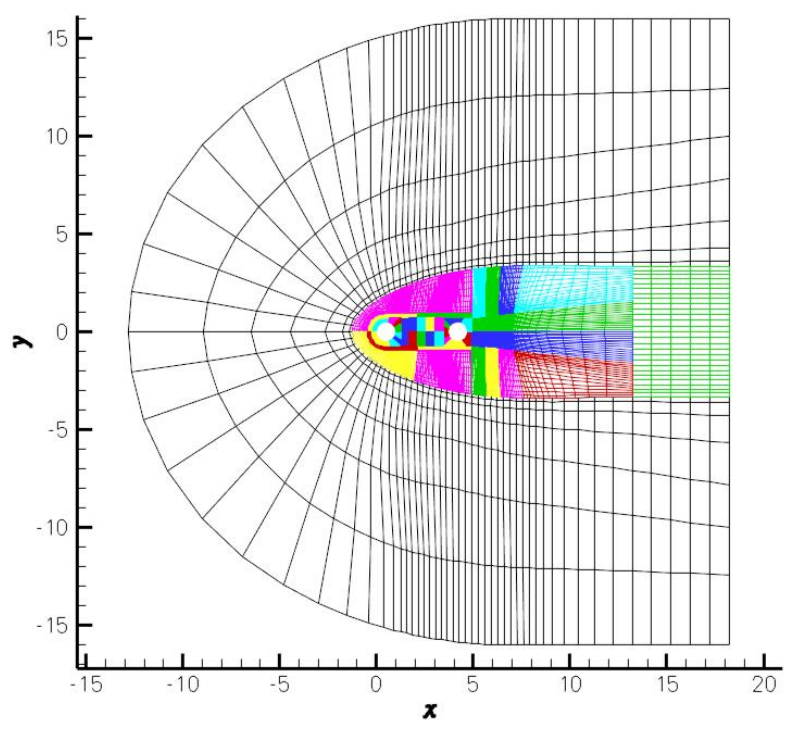

(b) Full grid

Figure 7. Planar view of grid (every 4th point).

conditions were imposed in the spanwise direction. We have not attempted to resolve any of end effects that are present in the experiments. The resolution of the spanwise direction was 97 points for a span of 3. Spanwise extents of 3 and 6 have been examined on the fine grid. This family of grids is denoted as Fxyz, signifying fine resolution in all 3 coordinate directions. The total grid count for a span of 6 is 80 million, representing our finest resolution. Simulations based on medium-level grids (derived from the finest grid by removing every other grid points in each direction) were performed to allow an assessment of the grid convergence of the computed solutions. This family of grids is denoted as Mxyz. Spans of 3, 6, and 18 have been examined, with 30 million grid points for the longest span. During the course of the work, we realized that although the planar resolution on the medium mesh was reasonable, the spanwise resolution did not seem to be sufficient. Therefore, a series of grids with the same spanwise resolution as the fine grid were generated. These grids denoted as MxyFz have a nearly isotropic cell spacing in the wake region between the cylinders. Again, spanwise extents of 3,6, and 18 were examined, with 60 million grid points for the longest span.

A constant non-dimensional time step of $t c_{\infty} / D=0.051$ or $t U_{\infty} / D=0.0855$ (corresponding to approximately 520 time steps per period for the primary shedding frequency) was used for all simulations. Limited calculations were also performed with double and half the time step without any appreciable differences in the first and second moment statistics, although the required effort to obtain sufficient samples makes it problematic to be definitive about such statements. The code employs a dual time-stepping algorithm with subiterations used to converge the solution within each time step. We nominally used 20 subiterations per time steps, but the number varied slightly for the different grids. CFL3D outputs the subiteration residual, lift, and drag after each subiteration, and we chose the number of subiterations to insure that the lift and drag plateaued within each time step and the residual dropped by 2 to 3 orders of magnitude. However, the convergence of the turbulence quantities was always worse than for the flow equations.

The simulation procedure includes several steps. First, a steady-state computation was used to set up the basic mean flow, followed by an unsteady calculation with random suction and blowing applied to different spanwise and azimuthal sections of both cylinder surfaces in order to accelerate the onset of 3D, unsteady flow structures. The forcing did not exceed 3\% of the freestream velocity. The forcing was turned off after shedding was observed, typically 200 time steps into the unsteady calculation. The simulations were then run for 5000-10,000 time steps to allow the transient flow field to wash out before collecting time records. Averaged flow quantities were produced by time-averaging over 20,00030,000 time steps $(10,000-15,000$ samples). The number of averages was increased by taking advantage of the spanwise homogeneity of the flow and spatial-averaging over the span.

Figure 8 compares instantaneous density contours from different resolution 3-D simulations. Previous 2-D results only captured the largest scales, whereas considerable detail is seen in the 3-D results. Particle image velocimetry (PIV) measurements from the BART experiments ${ }^{3}$ also exhibit the fine scales observed in the 3-D simulations. Reference 1 shows extensive comparisons between the simulations and the experimental PIV results. 


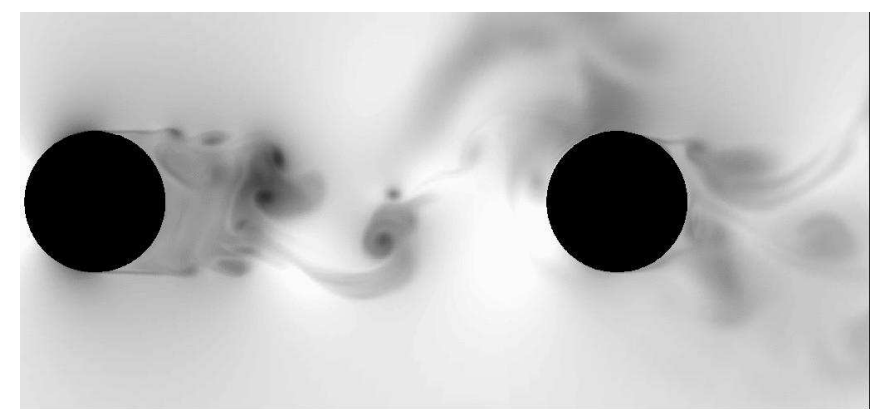

(a) Grid Mxyz, Span=3

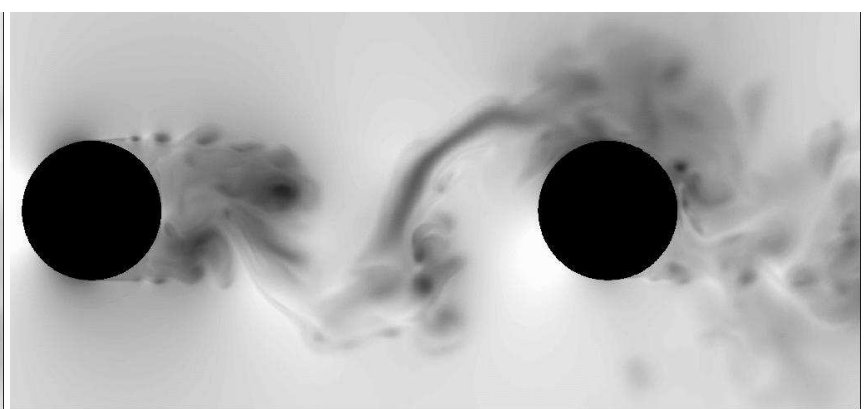

(b) Grid Fxyz, Span=3

Figure 8. Instantaneous density contours.

\section{III.B. Statistical Convergence of CFD Results}

Although both cylinders shed with a spacing of 3.7D, the shedding was intermittent and irregular. As can be seen in density contours in figure 8, Kelvin-Helmholtz type instabilities grow on the shear layers emanating from the cylinders. The eventual roll-up of the shear layers produces the primary shedding frequency, but the roll-up process was sometimes very intense, while nonexistent at other times. The history of the lift and drag coefficients as a function of time step iteration in figure 9 demonstrates this irregular behavior. Obviously, the significant low frequency component makes it very difficult to collect enough samples to obtain statistically significant results. The intermittent behavior can also be seen in the experimental surface pressure data to be shown later, and was one source of the experimental scatter. However, the experimental time record was typically a couple of orders of magnitude longer than what can reasonably be computed. Because of the nature of the phenomena under investigation and the limited time record available from $\mathrm{CFD}$, we assessed the computational results as the solutions evolved in time. Including additional time steps in averages of the CFD data always had a discernible affect on the results, but we have verified that we have collected enough samples in the current calculations to insure the variations are cyclic and do not show any clear trends as more averages are included.

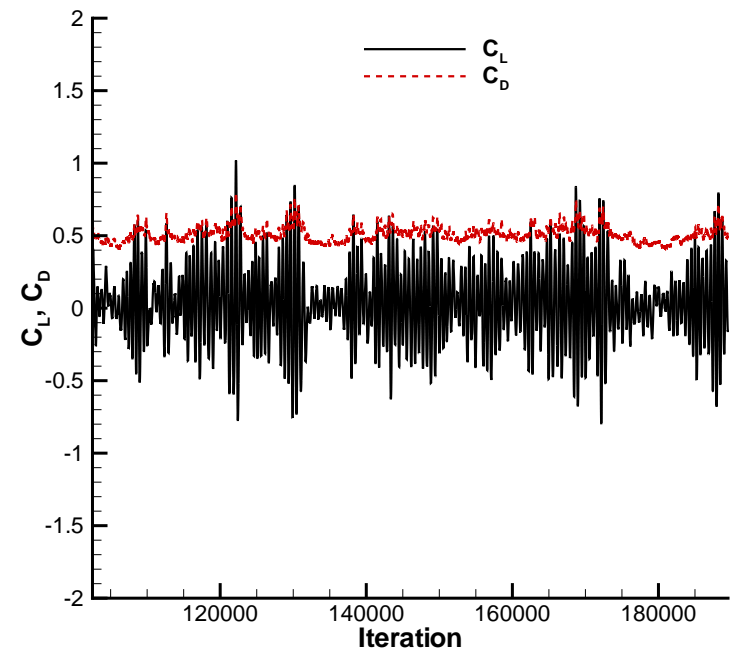

(a) Upstream cylinder

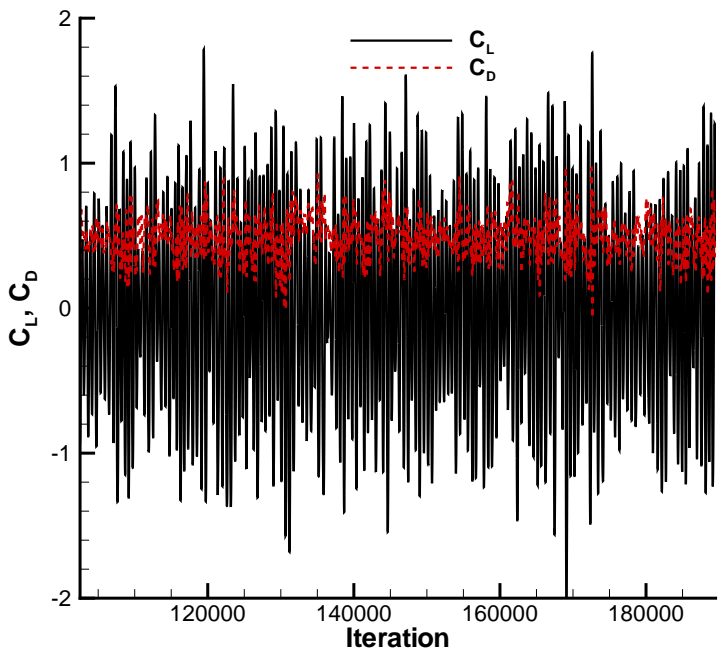

(b) Downstream cylinder

Figure 9. Unsteady lift and drag histories on the surface of the cylinders for the Mxyz, span $=3$ case.

To investigate the variation in the time-averaged quantities, cumulative averages were computed every 300 time steps using the Mxyz grid with a span of 3 and 6. These averages also include averaging along the span assuming homogeneity in that direction. Based on our observations, calculations with the coarsest grids and the shortest span lengths converge most slowly in time, presumably because there are fewer averages over the span. The sequences were started after the computation had been run long enough for a typical sampling period. For the span of 3, the sequence starts after 28,800 
unsteady time steps of sampling. For the span of 6 , the sequence starts after 17,400 time steps of sampling. The results for the span of 3 are shown in figure 10 and in 11 for the span of 6 . Because the average after every 300 time steps is plotted, the black and red bands represent the range over which the time average varies for the upstream and downstream cylinders, respectively. The variation is slightly greater for the shorter span. For $C p$, the range is quite small. In the $C p_{r m s}^{\prime}$ plots, the average at the beginning of the sequence is shown in green and the last one in blue. The variation is cyclic and does not exhibit any consistent trend with time. Although the variation for $C p_{r m s}^{\prime}$ is more significant than for $C p$, it is still quite small compared with the discrepancy with the experimental data. Furthermore, the variation is of similar magnitude to the experimental scatter seen in figure 4(b).

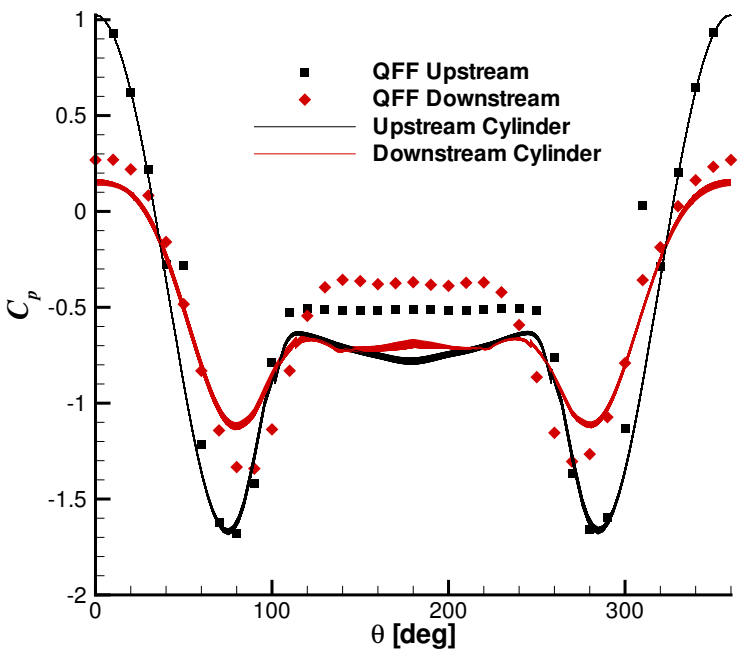

(a) $C p$ convergence

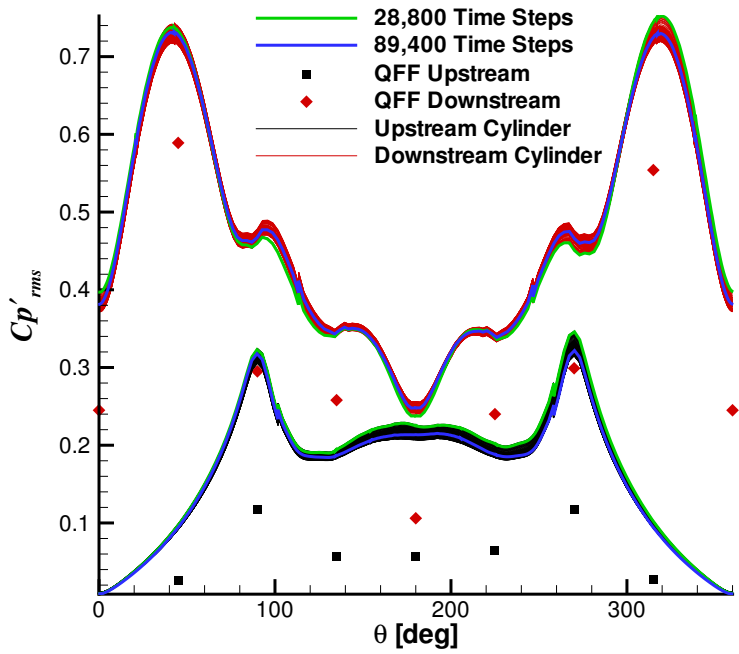

(b) $C p_{r m s}^{\prime}$ convergence

Figure 10. Convergence with time step of $C p$ and $C p_{r m s}^{\prime}$ for the Mxyz grid with a span of 3 . An average after every 300 time steps is plotted in black and red.

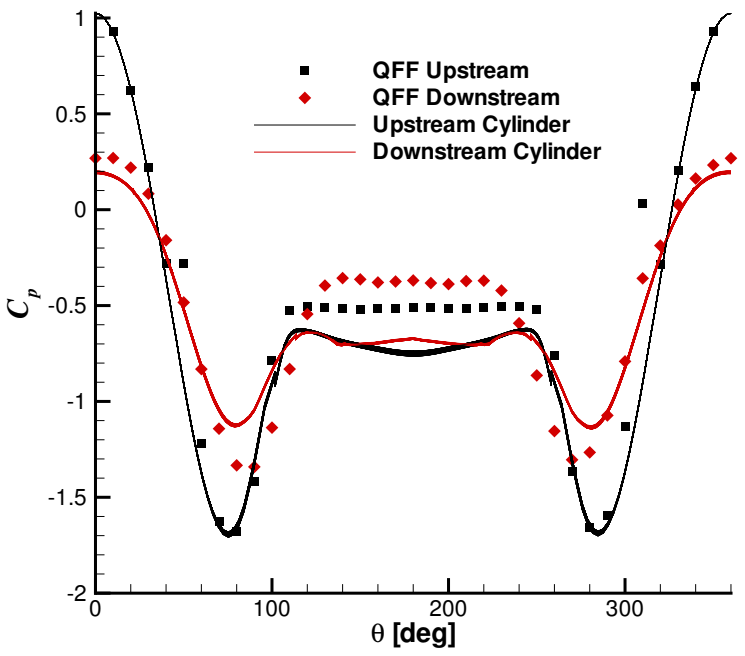

(a) $C p$ convergence

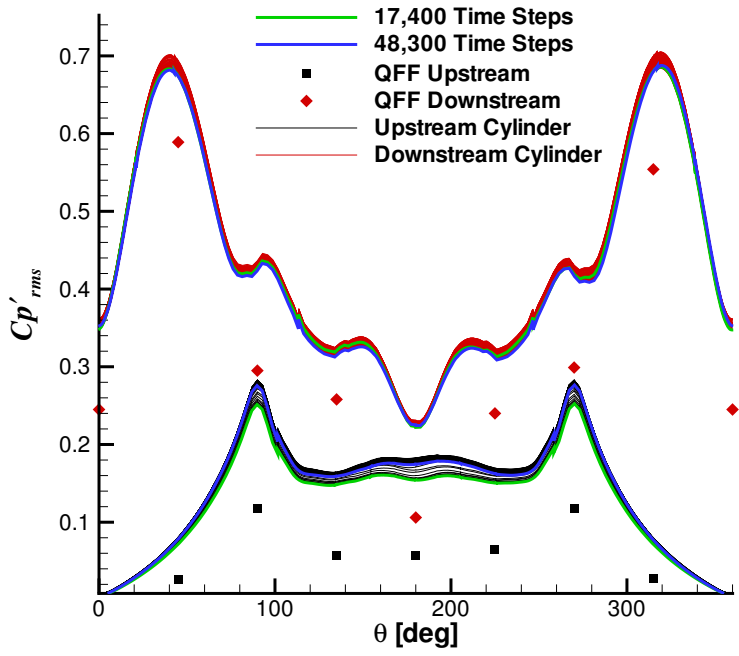

(b) $C p_{r m s}^{\prime}$ convergence

Figure 11. Convergence with time step of $C p$ and $C p_{r m s}^{\prime}$ for the Mxyz grid with a span of 6 . An average after every 300 time steps is plotted.

Figure 12 presents a similar sequence for the time-averaged mean velocity in the streamwise direction along the centerline between the two cylinders. PIV results from the BART experiment ${ }^{3}$ were used to obtain the experimental result. The temporal variation in the computed mean velocity is greater than was seen for the mean pressure on the 
cylinder surfaces, but the excursions are still only a few percent of the nominal value. Again, the variations are cyclic without any consistent trend.

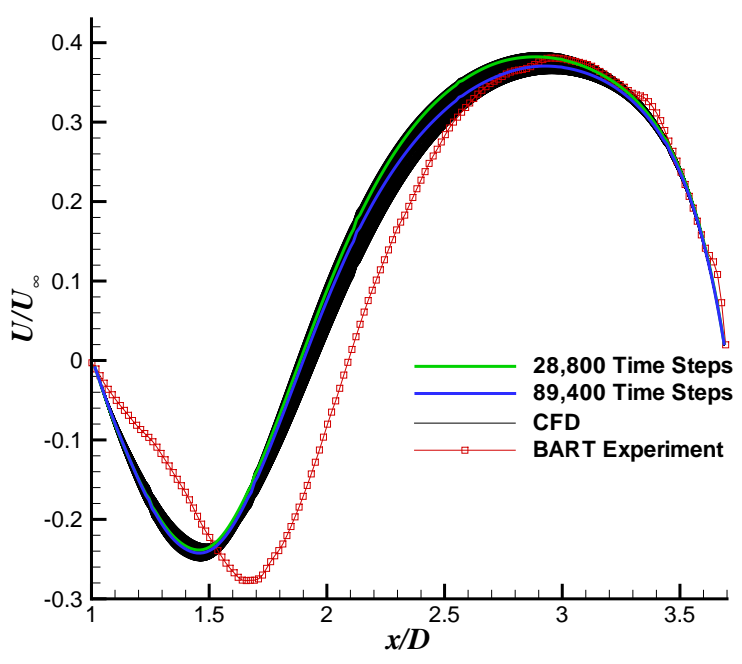

(a) $\mathrm{Span}=3$

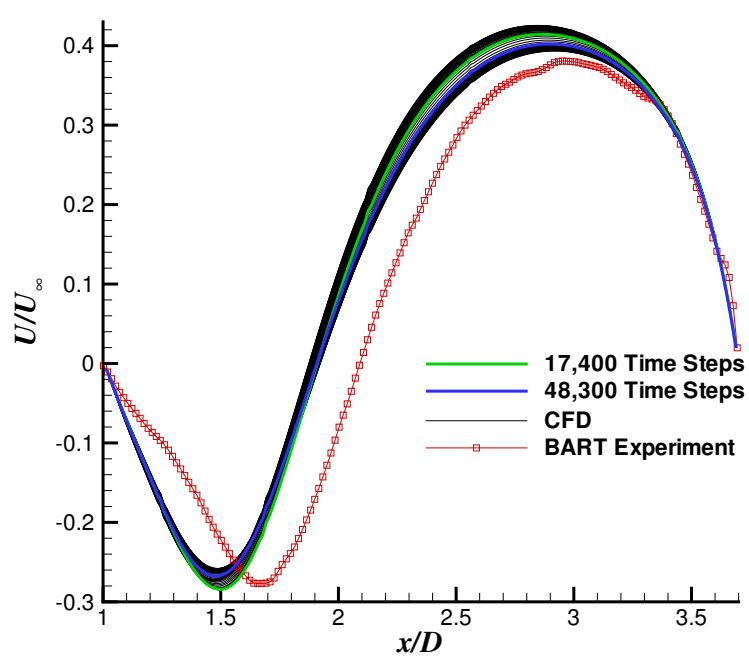

(b) $\mathrm{Span}=6$

Figure 12. Convergence with time step of the mean velocity along $y=0$ between the cylinders for the Mxyz grid. An average after every 300 time steps is plotted. Experimental values are from the BART experiment. ${ }^{3}$

Given the expected variability in the time-averaged results, we can make an initial assessment of the differences between solutions on different grids. Figure 13 presents the results for the surface pressure coefficient from the solutions showing the greatest difference. The results for all of the grids and span lengths are bracketed by the two plotted solutions. Overall, one might conclude from the $C p$ results that all of the solutions are similar and doing an equally good job of matching the experimental data. However, the picture is different for the mean velocity where there is significant and statistically meaningful variation between the solutions. The best match with the experimental data is from the Mxyz calculation with a span of 18, as seen in figure 14(a). From this plot, the span length appears to be the dominant parameter in matching the experimental data. However, figure 14(b) shows that the MxyFz result for a span of 18 has the greatest discrepancy with the experimental data. On the Fxyz grid, the solution also diverges from the experiment as the span increases. Clearly, the necessity of examining multiple quantities and multiple solutions is apparent.

\section{Results}

\section{IV.A. Surface Pressure}

Figures 15 and 16 present the $C p$ and $C p_{r m s}^{\prime}$ results for the longest spanwise extent used at each of the 3 grid resolutions. Figure 15(a), shows that the base pressure on the upstream cylinder from the MxyFz grid matches slightly better with the QFF experimental data; whereas, the Mxyz and Fxyz results match better with the BART ${ }^{3}$ data. As the simulations were performed in free air, one might expect that the CFD should match the open-jet QFF results. On the downstream cylinder, there is very little difference between the various computed solutions, but the discrepancy with experiment is considerably larger.

The variations in $C p_{r m s}^{\prime}$ between the different grids are more substantial. On the upstream cylinder (figure 16(a)), the fluctuations on the MxyFz grid are considerably lower than on the other two grids and match extremely well with the experimental data. However, the rms value only gives an impression of the overall energy in the fluctuations. As will be seen in the surface pressure spectra, the distribution of energy between the tone at the shedding frequency and the broadband component is not as favorable as the rms levels suggest. Also, the levels on the finest grid Fxyz are the highest. For a fixed span length, the Mxyz grid produces the highest levels, and the MxyFz grid produces the lowest. The finest planar grid resolves the shear layer much better than the medium grid allowing for much more rapid instability growth, and more intense fluctuations. Increasing the span, or increasing the spanwise grid resolution, gives the solution more freedom to develop three-dimensionally which has a tendency to reduce fluctuation levels. The results in figures 15-16 show that there is a complex interaction between the grid resolution and spanwise extent. 


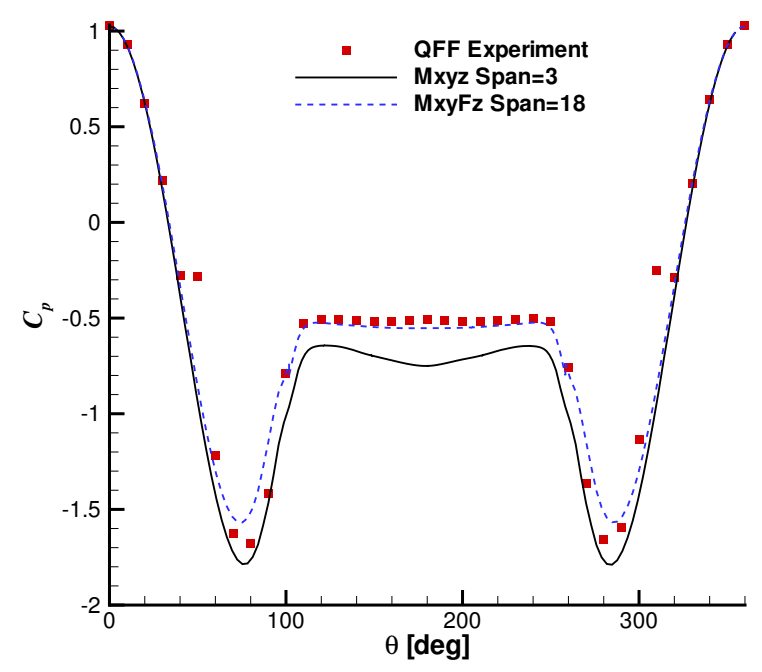

(a) Upstream cylinder

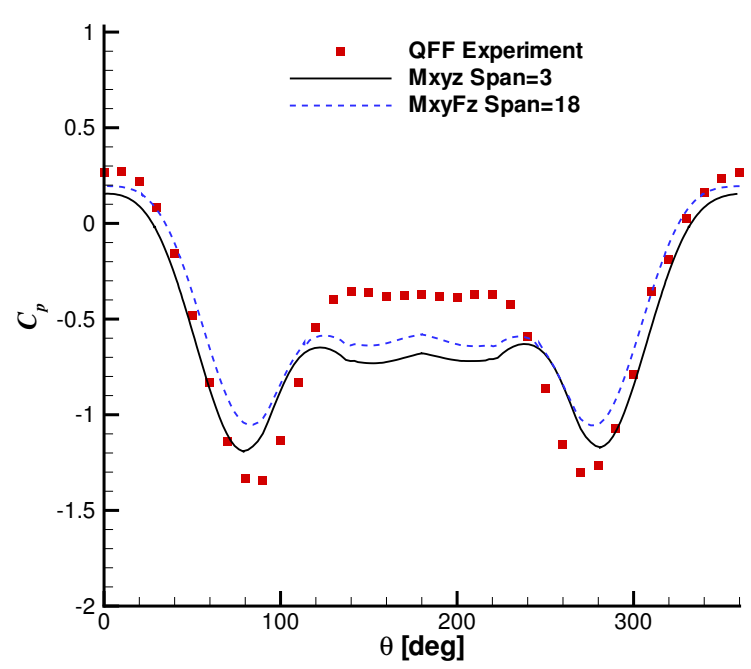

(b) Downstream cylinder

Figure 13. Comparison of the surface pressure coefficient from the computed solutions with the greatest variation.

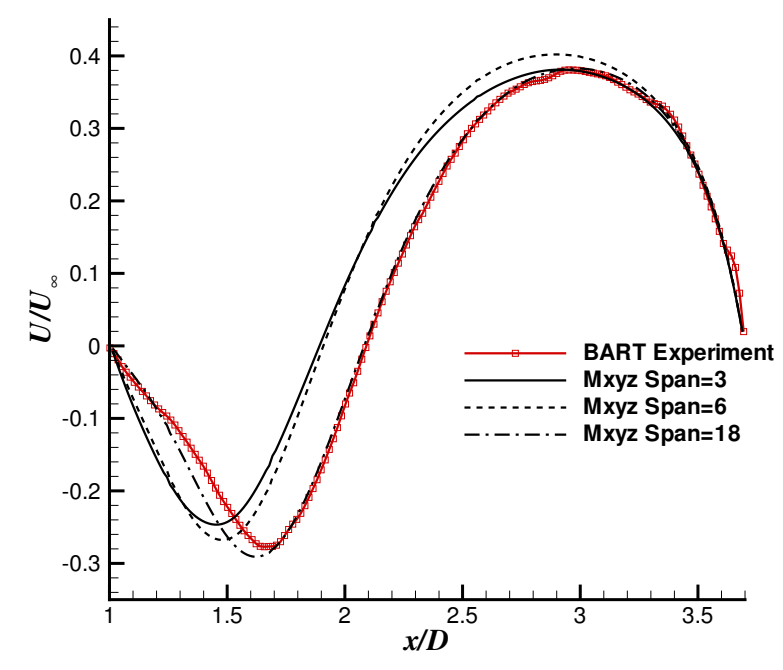

(a) Grid Mxyz

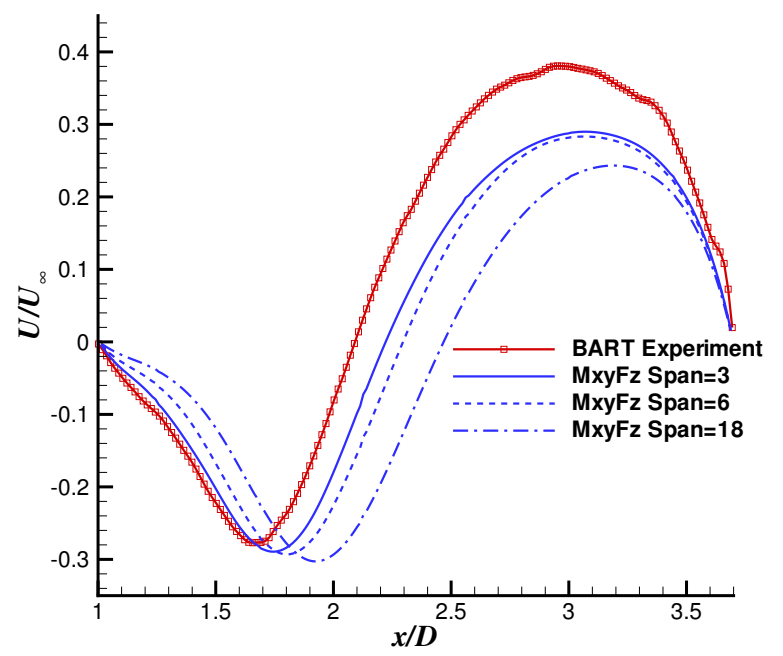

(b) Grid MxyFz

Figure 14. Comparison of mean velocity between the cylinders along $y=0$. Mean velocity experimental data are from the BART. ${ }^{3}$ 


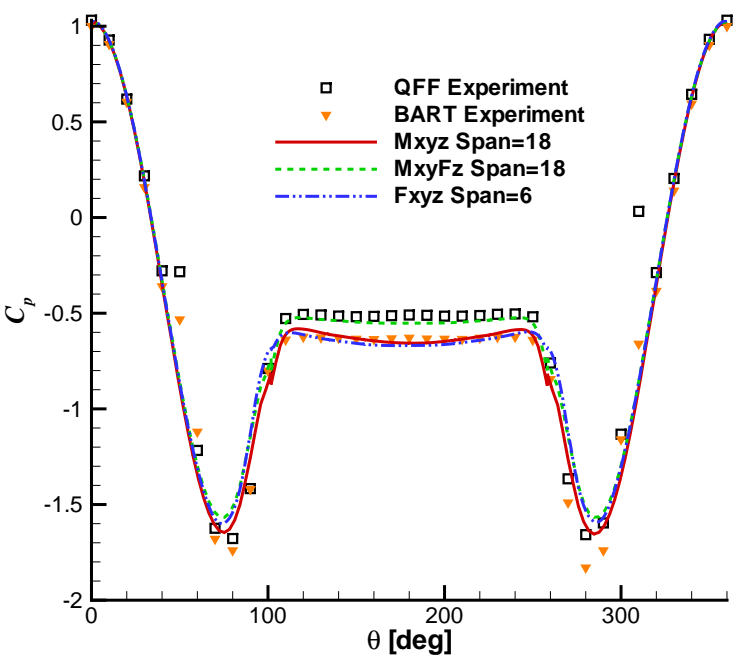

(a) Upstream cylinder

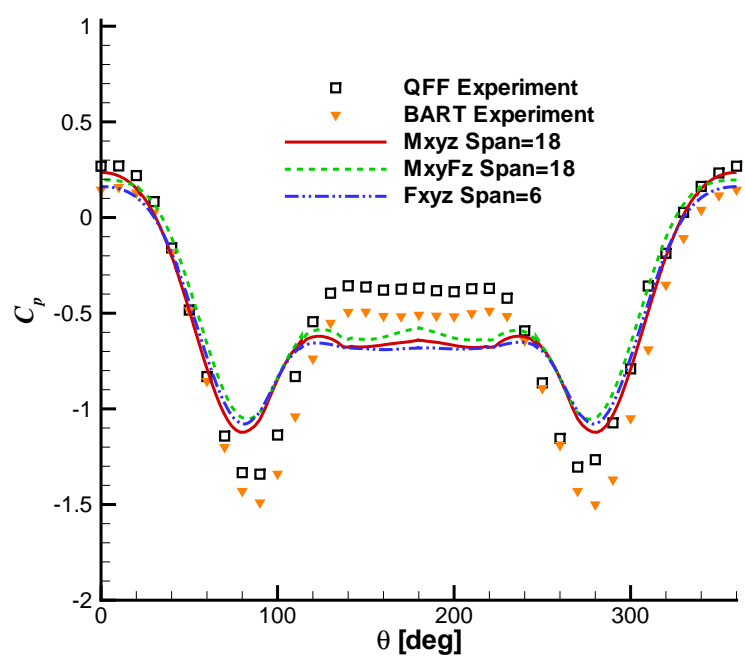

(b) Downstream cylinder

Figure 15. Coefficient of pressure on the surface of the cylinders.

Note that the range for the downstream cylinder in figure 16(b) is much larger than for the upstream cylinder. In general, the computations pick up the location of the peak fluctuations around $45^{\circ}$. This peak is caused by the impingement of the wake on the downstream cylinder. The flow separates at the second peak, which is around $135^{\circ}$ in the experiment, but is closer to $100^{\circ}$ in the computations. The ability of the CFD to predict delayed separation in the presence of high turbulence shows that some of the physics is being captured. The CFD solutions also show a plateau or third peak around $150^{\circ}$, but this is not borne out in the experiments. Although the fluctuation levels on the downstream cylinder dominate, they are in a sense a reflection of what occurs on the upstream cylinder. Higher levels on the upstream cylinder will produce higher levels on the downstream cylinder because of the wake interaction. However, the process is nonlinear, so there is not a one-to-one correspondence.

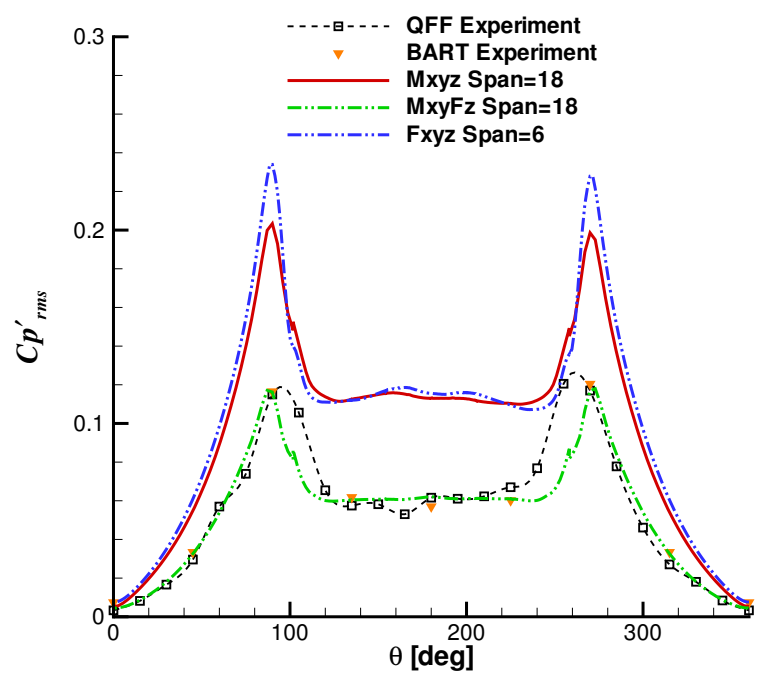

(a) Upstream cylinder

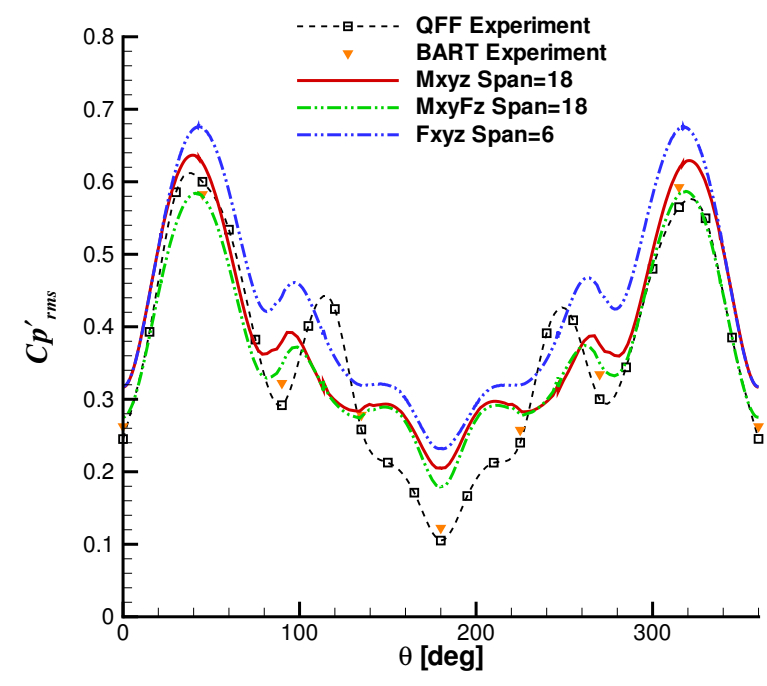

(b) Downstream cylinder

Figure 16. RMS of the coefficient of pressure on the surface of the cylinders. 


\section{IV.A.1. Spanwise Refinement and Extent}

Results for a standard grid refinement for a spanwise length of 3 have already been presented in our previous work. ${ }^{1}$ Figure 17 presents the results of a spanwise refinement for the medium planar grid. The Mxyz grid has a spanwise resolution of 49 points, and the MxyFz grid has 97 points in the span. We have performed a similar spanwise refinement using the fine planar grid. Although the details are different, all the trends are similar. The biggest changes occur between a resolution of 49 and 97 points. The rms levels drop significantly with the grid refinement, and changes in base pressure are also noticeable. The results seem to indicate that the spanwise resolution of 49 points is marginal, at best.

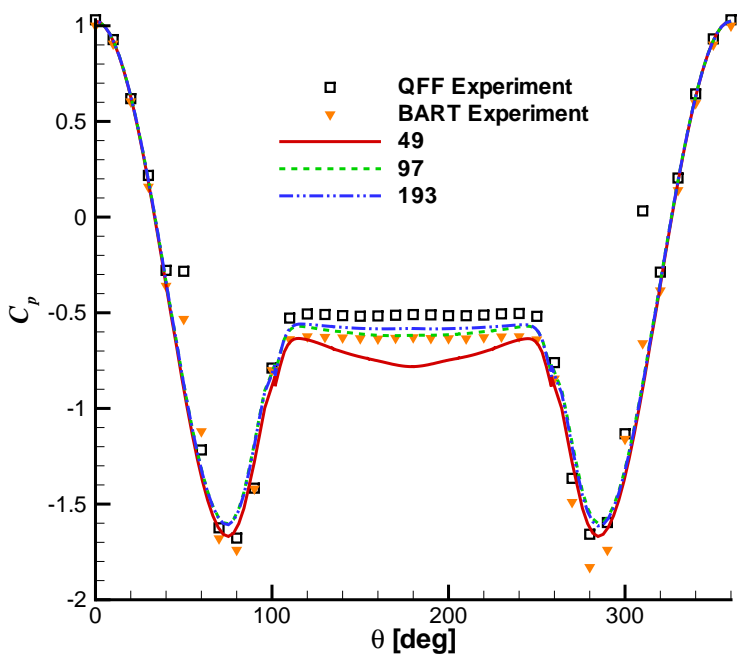

(a) Upstream cylinder

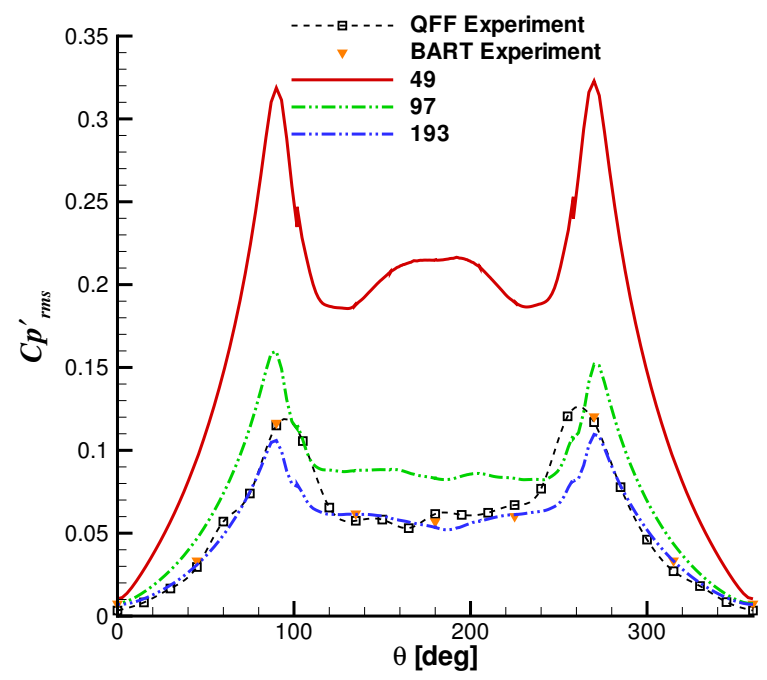

(c) Upstream cylinder

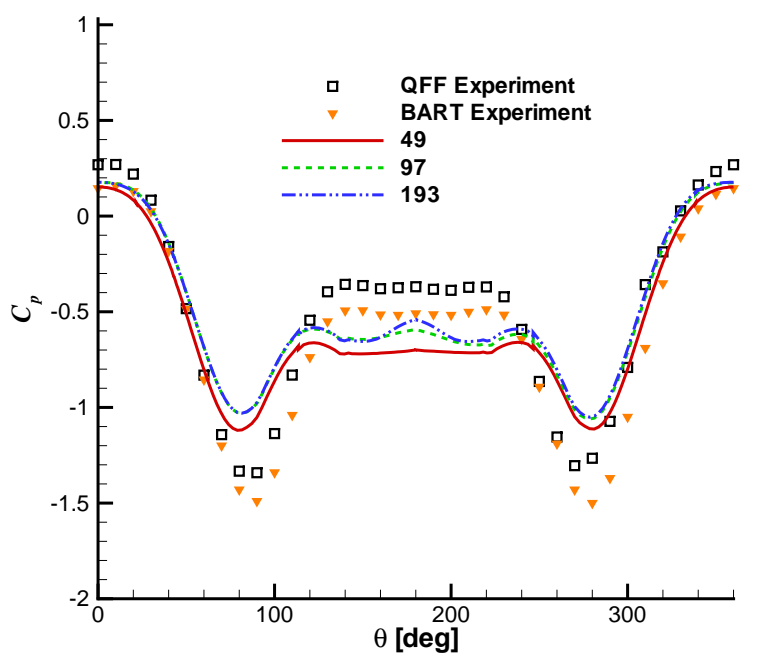

(b) Downstream cylinder

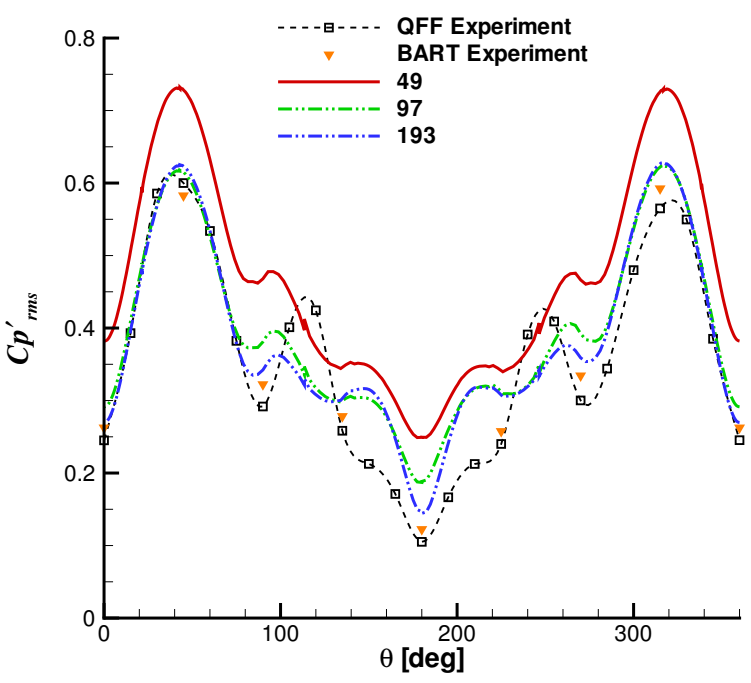

(d) Downstream cylinder

Figure 17. Spanwise refinement for a span of 3 with Mxy fixed. The Mxyz grid spanwise resolution is 49 points.

Because of limited computational resources, we have been unable to run a calculation on the Fxyz grid with a span of 18 which would require 240 million grid points. However, we have completed calculations with spans of 3, 6, and 18 for both the Mxyz and MxyFz grids. The results are presented in figures 18 and 19. The trends for both meshes are similar to that found for the grid refinement. Increasing the spanwise length increases the base pressure on the front cylinder and decreases the rms levels. Although the results for the spans of 6 and 18 for the MxyFz grid in figure 19 seem similar, there are some meaningful differences which will be brought out by the surface pressure coherences. 


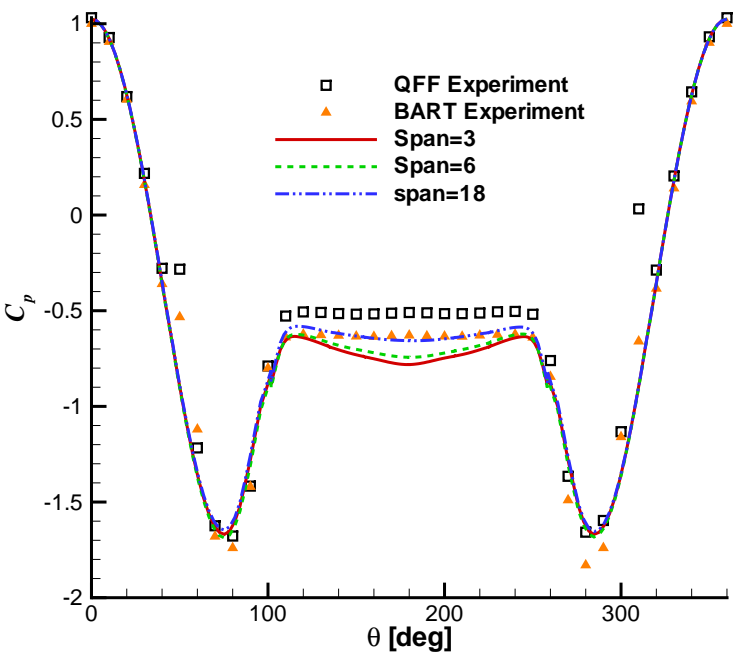

(a) Upstream cylinder

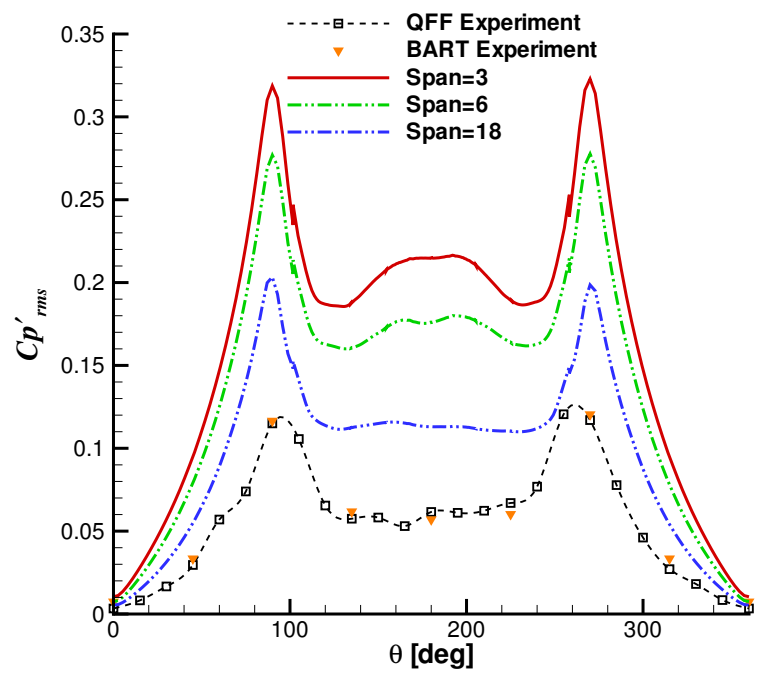

(c) Upstream cylinder

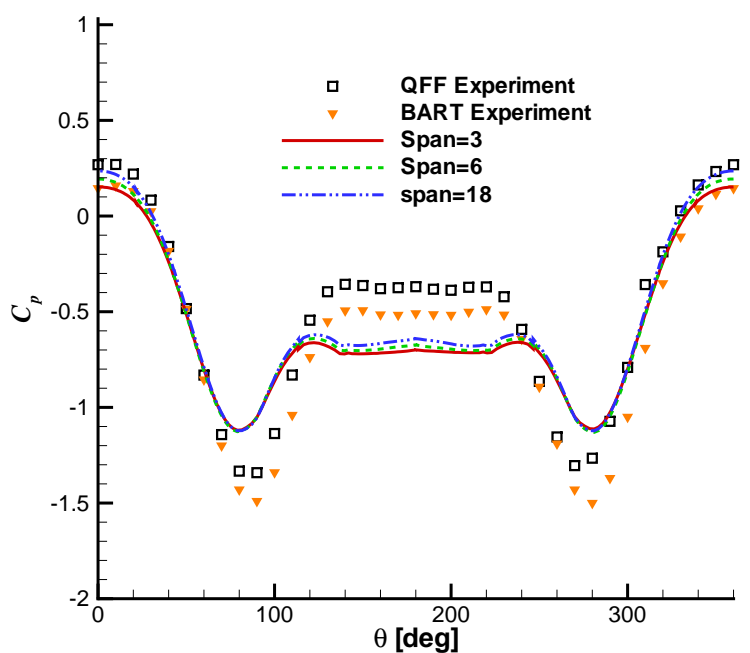

(b) Downstream cylinder

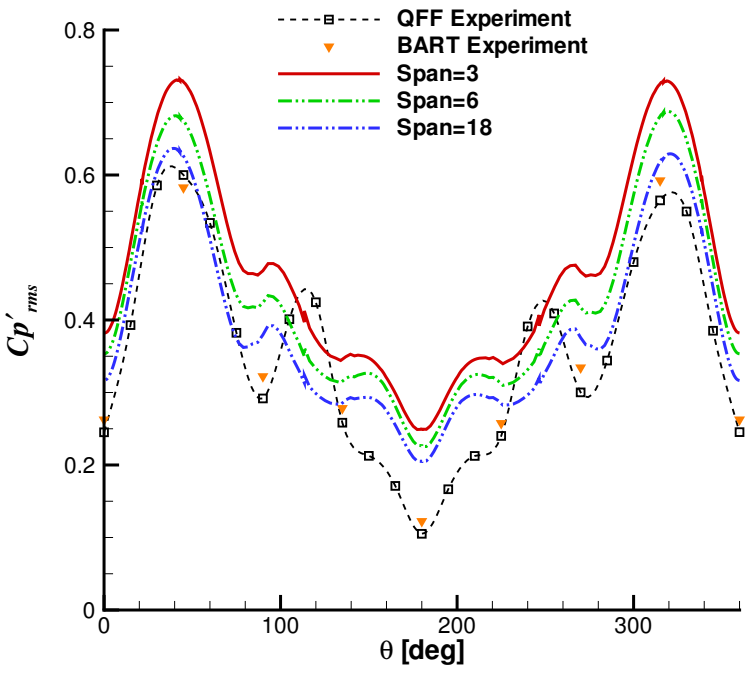

(d) Downstream cylinder

Figure 18. Variation with spanwise extent for Mxyz grid. 


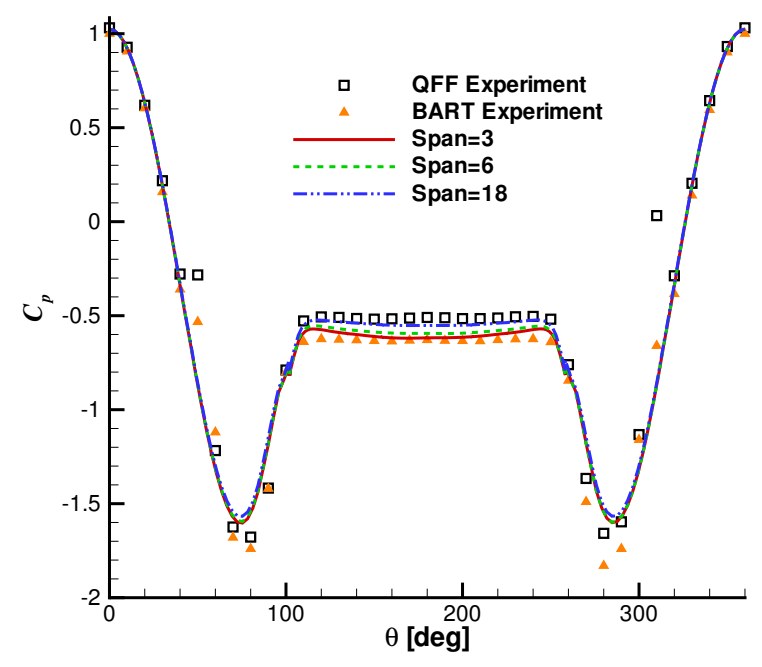

(a) Upstream cylinder

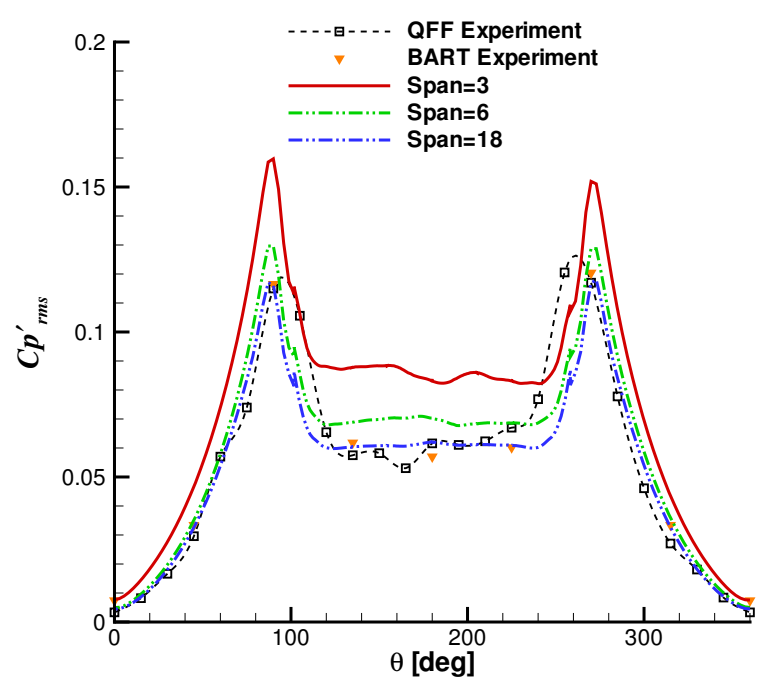

(c) Upstream cylinder

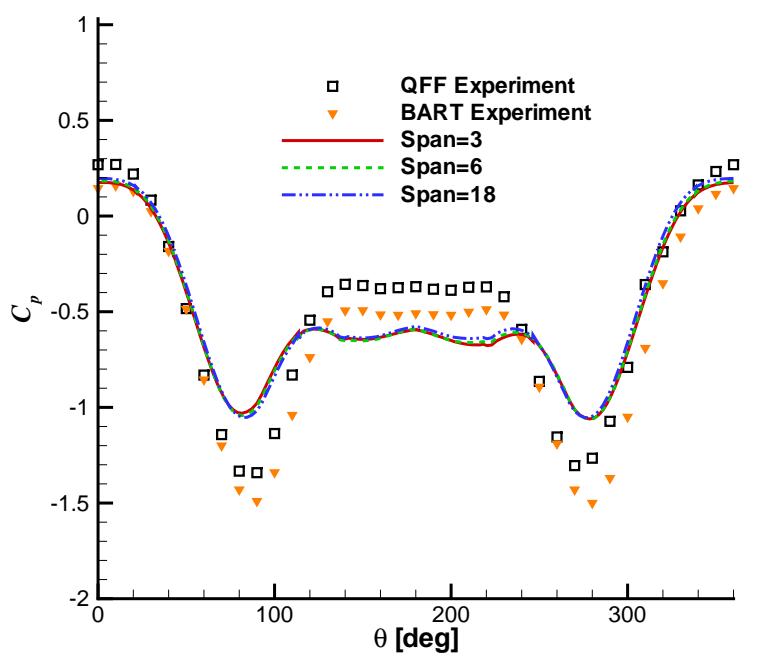

(b) Downstream cylinder

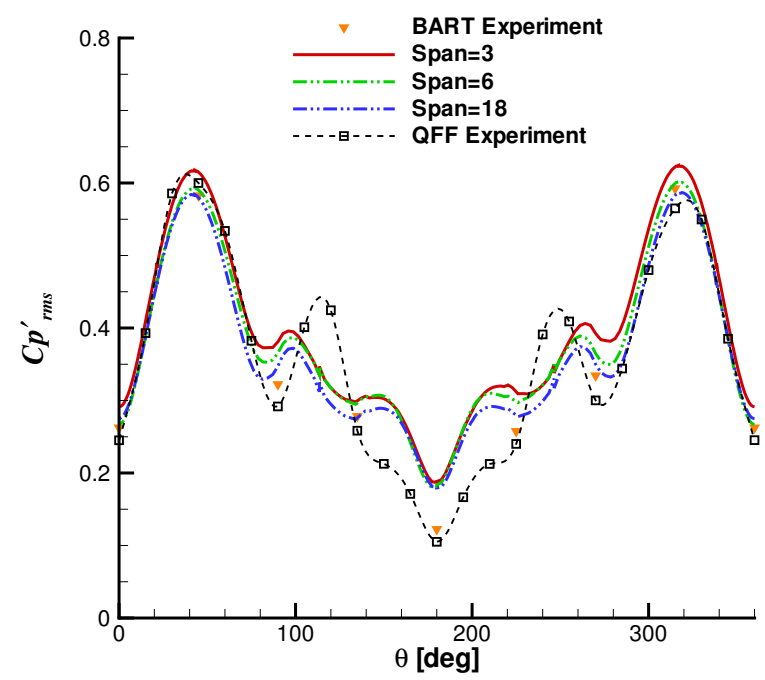

(d) Downstream cylinder

Figure 19. Variation with spanwise extent for MxyFz grid. 


\section{IV.B. Surface Pressure Spectra}

One can get a much more detailed picture of the surface pressure fluctuations by looking at the spectra. Figure 20 compares the results from the Mxyz calculation with the experiment. The results are presented as power spectral density (PSD). To account for the different freestream velocities in the experiment and computation, the CFD pressures were corrected to the experimental condition by scaling them by the square of velocity ratio $(0.1274 / 0.166)$. The frequency was Strouhal scaled, which results in multiplying the frequency by the velocity ratio. The CFD time record was divided into segments of 4096 samples (8192 time steps) with an overlap of 50\%. A Hamming filter was applied to each segment. The results were also spanwise averaged. The bin width is $14.28 \mathrm{~Hz}(10.96 \mathrm{~Hz}$ after scaling), compared with $3.125 \mathrm{~Hz}$ for the experimental data. Figure 20(a) shows a time history of the signal on the upstream cylinder at $90^{\circ}$. Both the QFF experimental data and the CFD result, from the MxyFz grid with a span of 18, show the primary shedding frequency superimposed on and modulated by low frequencies. Also, there are time segments when the primary shedding is suppressed (0.08-0.1 s). The time record in the figure includes all of the CFD data, but only about 1/100th of the experimental data. Hence, the experimental data can be processed at a much smaller bin width and with many more averages. However, the bin width has an effect on such signals. Figure 20(b) compares the power spectral density for the experimental data when processed with 3.125 and $12.5 \mathrm{~Hz}$ bin widths with the CFD result processed at $14.28 \mathrm{~Hz}$ (scaled to $10.96 \mathrm{~Hz}$ ). Clearly, a larger bin width reduces and broadens the tonal peak, but has little effect on the rest of the spectrum. Furthermore, the experimental scatter between different runs is around $2 \mathrm{~dB}$ at the tone. Hence, one must be careful when making comparisons to account for processing nuances, and not expect exact agreement at tones.

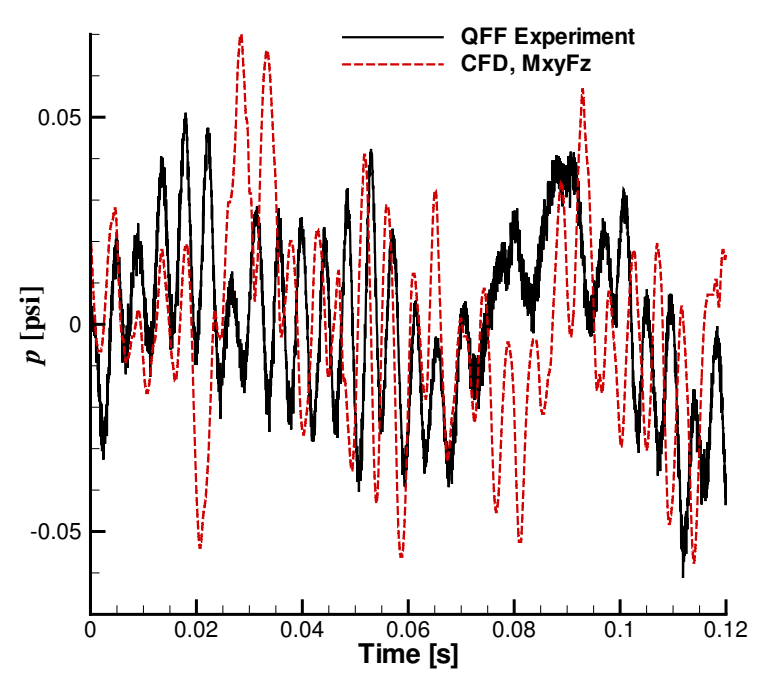

(a) Time History

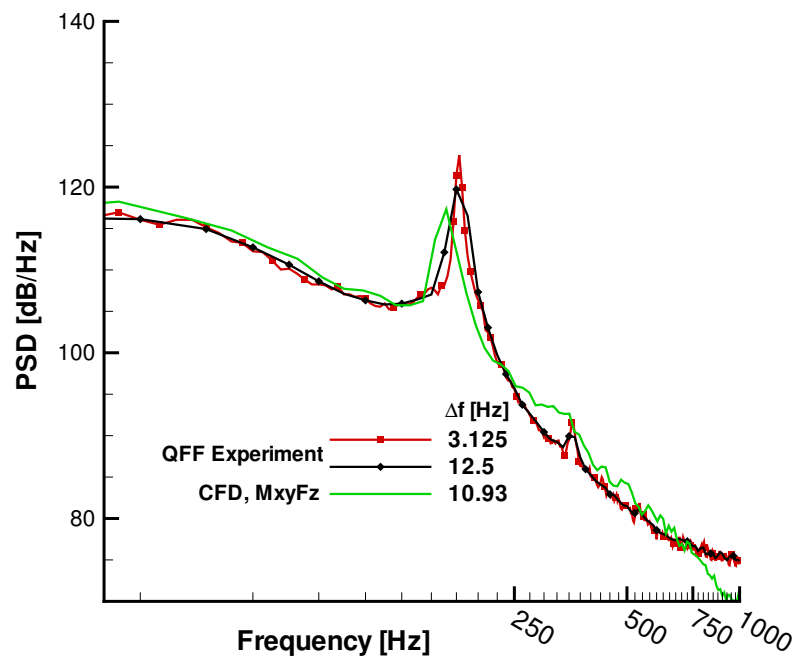

(b) Spectra

Figure 20. Surface pressure time history and spectra on the upstream cylinder at $\theta=90^{\circ}$ with the experimental data processed with different bin widths. The CFD results are for a span of 18.

Figure 21 presents the surface pressure spectral results for the Fxyz grid and a span of 6 . Only the results for $90^{\circ}$ to $180^{\circ}$ are presented as that covers most of the fluctuation energy. Overall, the spectral shapes are similar, although the amplitudes are somewhat different. On the front cylinder, both the primary tone and the broadband levels are higher than the experimental values. In the experimental data, the 2 nd harmonic has a pronounced peak at all three angles, but there is only a barely discernible hump for the CFD. Beyond $1 \mathrm{kHz}$, the experimental data at $90^{\circ}$ and $135^{\circ}$ show some increases with frequency that are not present in the CFD. Jenkins et al. ${ }^{3}$ speculate that these features are caused by spanwise rollers generated by the trips. For all angles, there is considerable energy in the low frequencies. The plots start at $20 \mathrm{~Hz}$, and there is nearly as high a value of the spectral density at that frequency as at the tonal peak, indicative of the intermittent nature of the shedding on the upstream cylinder.

Figure 21(b) shows that the levels on the downstream cylinder are around $15 \mathrm{~dB}$ higher than on the front cylinder. Results for $0^{\circ}$ to $90^{\circ}$ are presented as that is where the wake interaction and highest levels occur. However, the levels at the lowest frequencies are now considerably lower than the tonal peak. Nonetheless, these levels are still similar to the peak levels on the upstream cylinder. Furthermore, the CFD is slightly lower at the tones, but still has higher broadband levels. The spectral shapes are again quite similar. At $0^{\circ}$ the second harmonic is quite pronounced. The impacts of 
the wake from the upstream cylinder on the downstream cylinder are centered around $\pm 45^{\circ}$ and occur at the shedding frequency. The $0^{\circ}$ point equally feels the influence of all of these impacts, which occur at twice the shedding frequency.

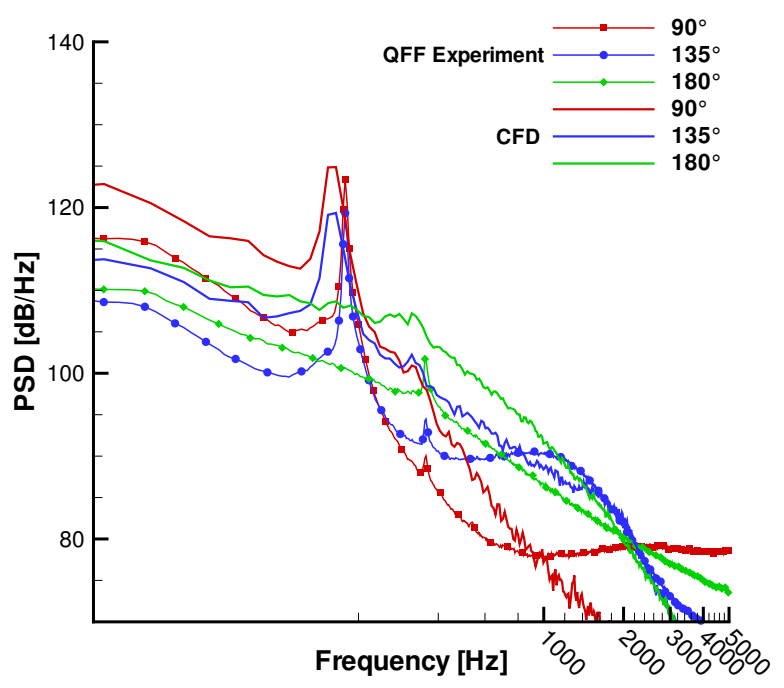

(a) Upstream cylinder

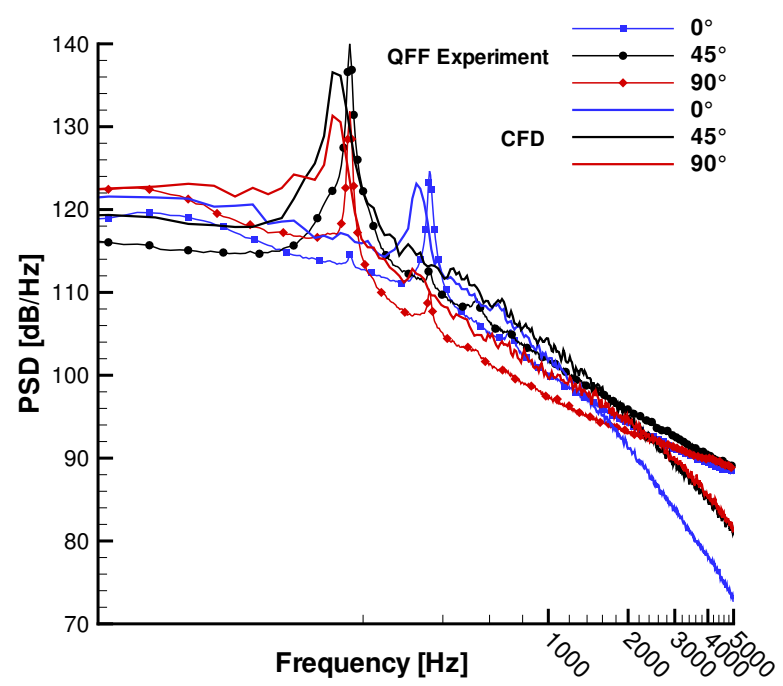

(b) Downstream cylinder

Figure 21. Surface pressure power spectral density for Fxyz grid with a span of 6.

Similar spectral results for the MxyFz grid with a span of 18 are presented in figure 22. Compared with the fine grid results, all of the levels are lower, especially at the tone on the upstream cylinder. Even taking into account the effect of the processing at different bin widths, the MxyFz results are probably underpredicting the levels at the tone even though the rms levels are similar to the experimental values.

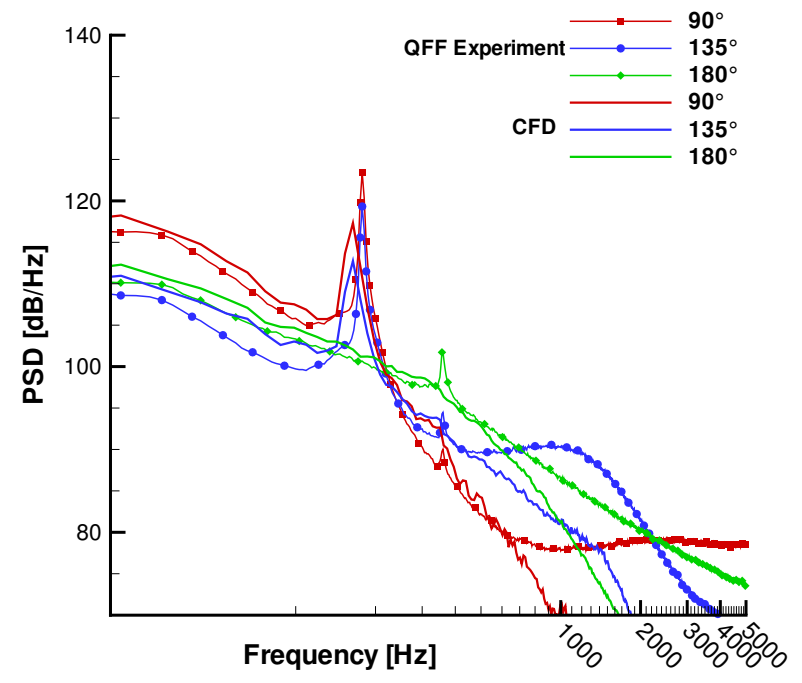

(a) Upstream cylinder

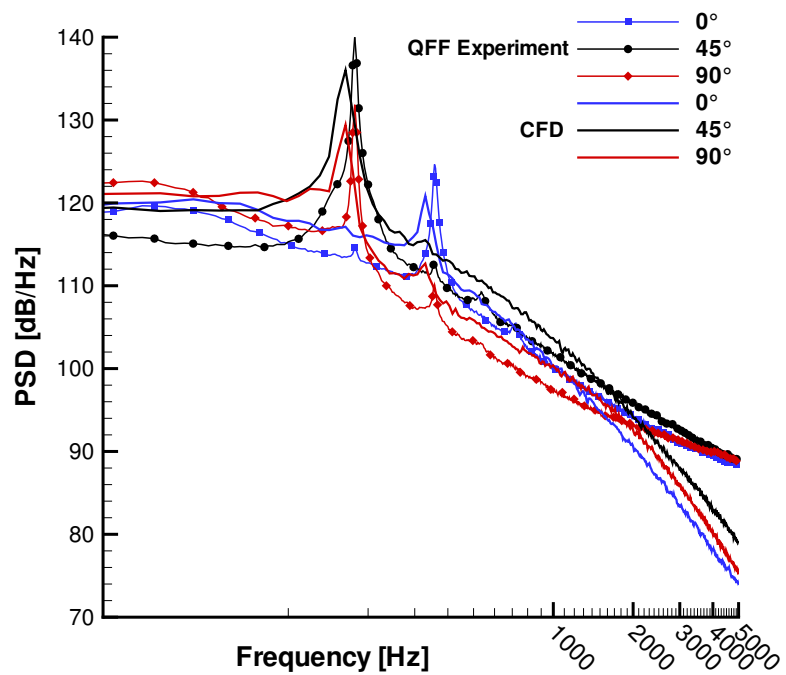

(b) Downstream cylinder

Figure 22. Surface pressure power spectral density for MxyFz grid with a span of 18.

In an attempt to better identify the shedding frequency from the CFD data, we processed each time trace using the entire time record to reduce the bin width. We still averaged the signal over the span. The bin width was around $4 \mathrm{~Hz}$. The results for each family of grids was independent of the span to within the resolution of the bin width. The results are presented in Table 1. Although there is little variation in the results, it is interesting that the trend is away from the experimental value as the grid is refined. 


\begin{tabular}{|c|c||c|c|c|}
\hline & Experiment & Mxyz & MxyFz & Fxyz \\
\hline \hline Frequency & $178 \mathrm{~Hz}$ & $166 \mathrm{~Hz}$ & $163 \mathrm{~Hz}$ & $157 \mathrm{~Hz}$ \\
\hline $\mathrm{St}$ & 0.232 & 0.219 & 0.215 & 0.207 \\
\hline
\end{tabular}

Table 1. Primary shedding frequency as measured on the front cylinder at $90^{\circ}$.

\section{IV.C. Correlation and Coherence}

Information about how well the simulations capture the spanwise variation can be ascertained from the correlation. Figure 23 compares the experimental and computational results at $135^{\circ}$. Because periodic boundary conditions are employed in the computations, only half of the span is relevant. Also, the CFD results are averaged over all grid point combinations with the same spanwise spacing. Although the average produces a smooth curve, there is quite a bit of scatter in the CFD data that increases with the separation distance. Similar scatter is also present in the experimental data and has been documented previously. ${ }^{15}$ In general, the CFD correlations are somewhat lower than those in the experiment, with the discrepancy larger for the downstream cylinder. The correlations from the Fxyz computations on the shorter span of 6 never drop to the levels seen in the experiment or the other computations. Only about the 1-2 diameters of results for the span of 6 matches well. Even though no trip was employed on the downstream cylinder, the correlation is similar to that on the upstream cylinder. The correspondence could be an indication that the primary energy-containing eddies from the upstream wake do not decorrelate very much before impacting and influencing the signal on the downstream cylinder.

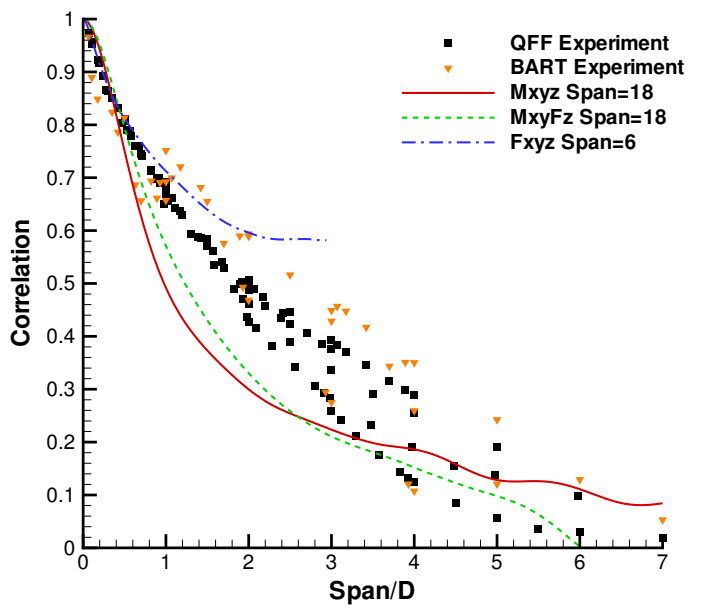

(a) Upstream cylinder

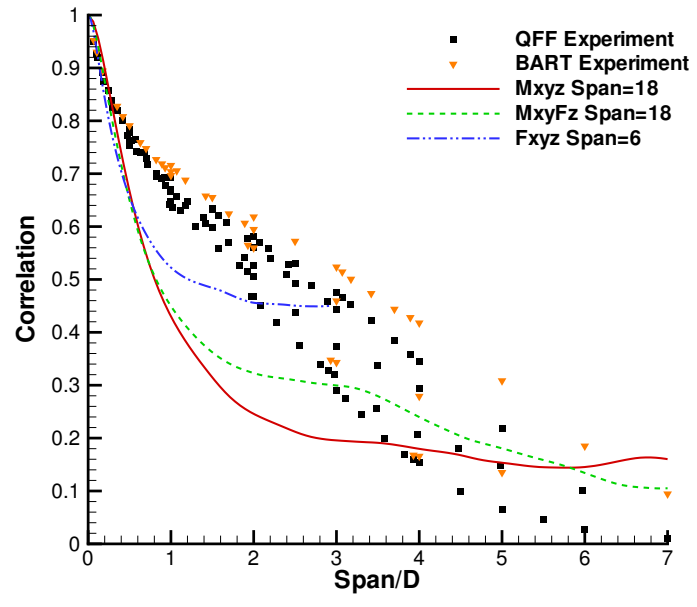

(b) Downstream cylinder

Figure 23. Correlation comparison at $\theta=135^{\circ}$.

More specific information about the spanwise uniformity in the flow can be acquired by using the coherence to isolate separate frequencies. In particular, the coherence at the primary shedding frequency is presented in figure 24 . The angle is $90^{\circ}$ as it is more indicative of the peak in the energy, but the results do not change much between $45^{\circ}$ and $135^{\circ}$. Both the CFD and experimental results were obtained using an energy weighted average of the coherence within $\pm 11 \mathrm{~Hz}$ of the shedding frequency. Without the averaging, the experimental results would be higher because of the much smaller bin width used in the processing which isolates the tone where the coherence tends to be highest.

Similar to the correlation data, the CFD results from shorter spans never see low levels of coherence. Also, regardless of the length of the span in the calculation, the coherence of the upstream and downstream cylinders decay similarly with spanwise separation distance. The trends in the results as the span was increased from 3 to 6 are different for the 3 grid resolutions, which may be an indication that the spanwise extent is too short. Counterintuitively, the calculation with increased grid resolution for the span of 18 is seen to have higher coherence. We had expected the finer grids to produce more three-dimensionality and thereby decorrelate faster.

Figure 25 presents the coherence at the shedding frequency obtained using the Fxyz grid and a span of 6 for angles between $0^{\circ}$ and $180^{\circ}$ on both the upstream and downstream cylinders. Experimental data for $0^{\circ}$ is not currently available. 
The plots show that the coherence is similar between the upstream and downstream cylinders, and the CFD results only follow the experiment up to 1 diameter. On the front cylinder, the coherence is very low at $180^{\circ}$ as one would expect for the completely separated region. On the downstream cylinder, the coherence is still low, but nonnegligible at $180^{\circ}$. The CFD picks up the trend, but underpredicts the change. Between $45^{\circ}$ and $135^{\circ}$, there is very little change in the coherence.

The results for the span of 18 calculation on the MxyFz grid are shown in figure 26. In general the agreement is considerably improved relative to the shorter span calculations. Although the CFD tends to underpredict the coherence for short separation distances, and overpredict it for longer ones, the results are within the uncertainty in the experimental and computational data.

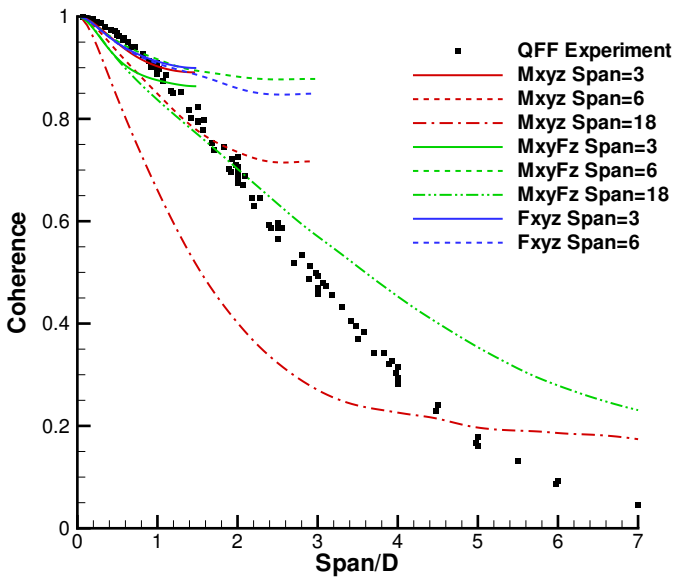

(a) Upstream cylinder

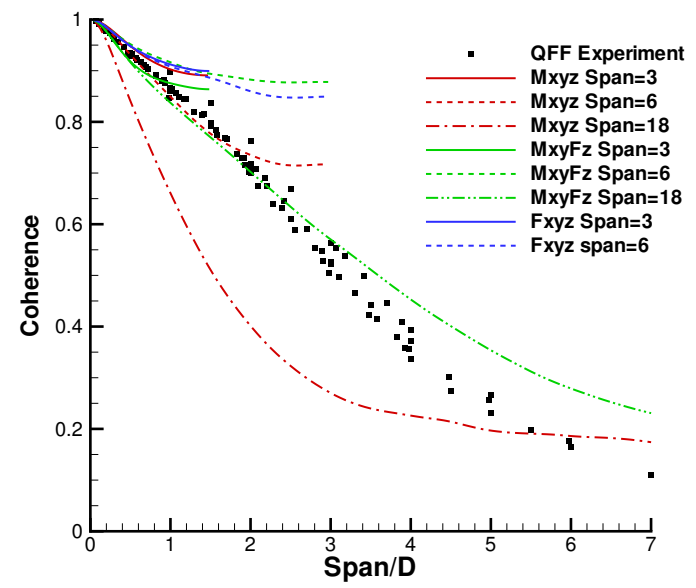

(b) Downstream cylinder

Figure 24. Coherence at $\theta=90^{\circ}$ of the primary shedding frequency.

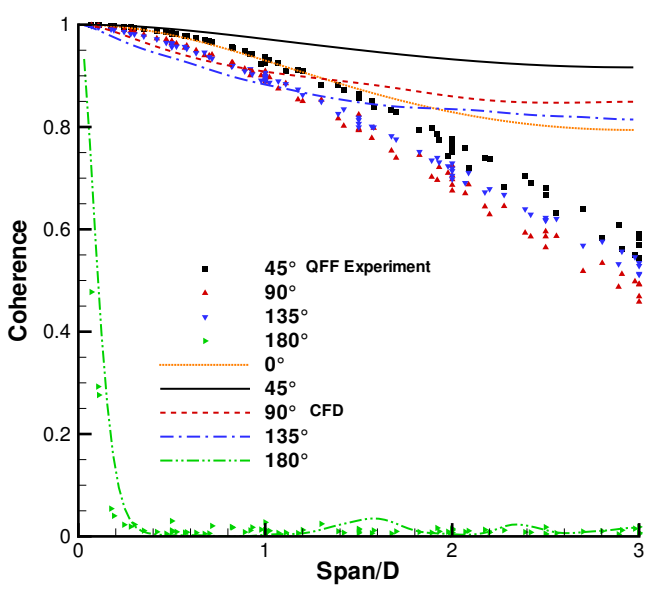

(a) Upstream cylinder

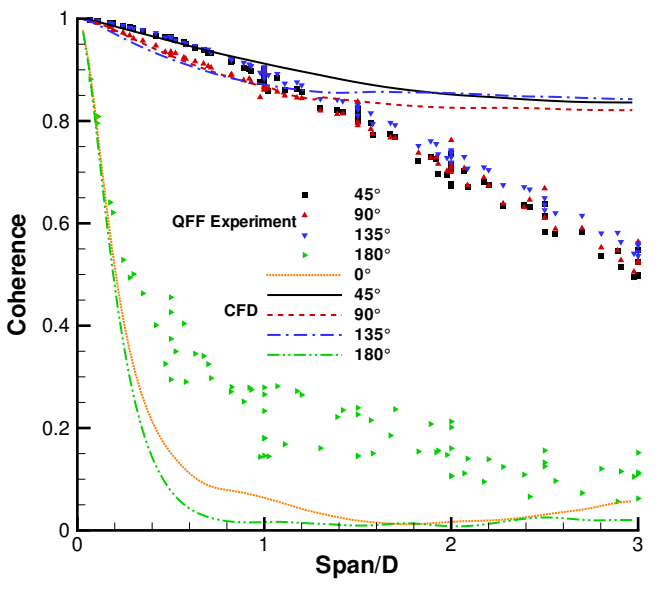

(b) Downstream cylinder

Figure 25. Coherence at $90^{\circ}$ of the primary shedding frequency for Fxyz, span=6.

The previous results have focused on the coherence of the primary shedding frequency as a function of spanwise separation. In figure 27, the coherence spectra for a separation distance of 0.18 diameters is presented. Peaks in the coherence can be seen in the shedding frequency and the 2 nd and 3 rd harmonics of the experimental data. The simulations miss the peak in the second harmonic, and the coherence on the upstream cylinder doesn't fall off with frequency as expected. The agreement on the downstream cylinder is much better at the higher frequencies. At this short separation distance, there is very little change between the CFD results on different grids. However, figure 28 shows that for a separation distance of 1 diameter, the coherence for the Mxyz grid is lower, especially at the peaks. The CFD results 


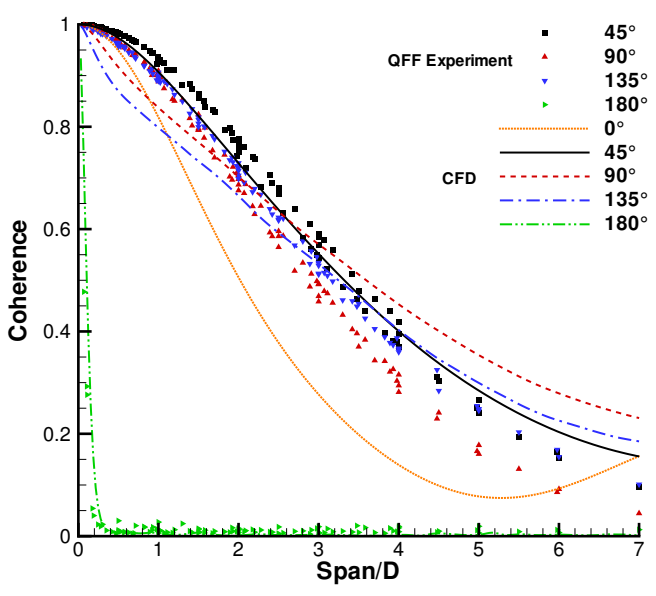

(a) Upstream cylinder

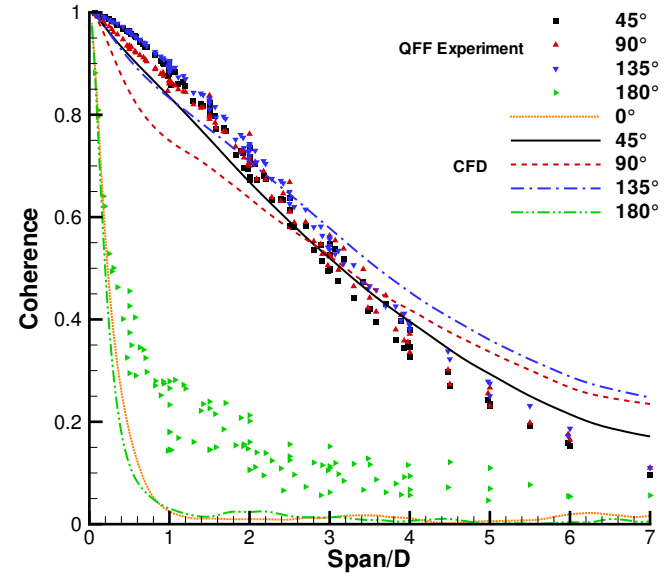

(b) Downstream cylinder

Figure 26. Coherence of the primary shedding frequency for MxyFz, span=18.

still show an enhanced coherence at high frequency, although the effect is diminished. The peak at the third harmonic is now difficult to identify even in the experimental data. Plots for larger separation distances look similar but with the peaks diminishing and less broad.

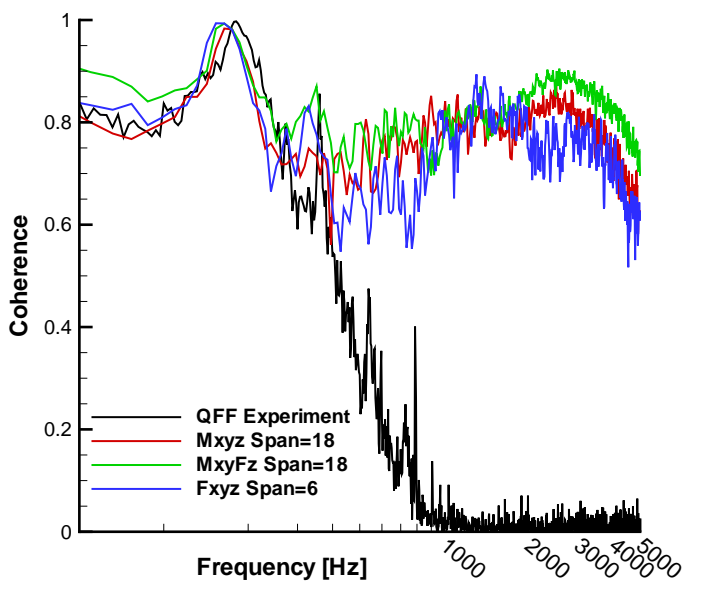

(a) Upstream cylinder

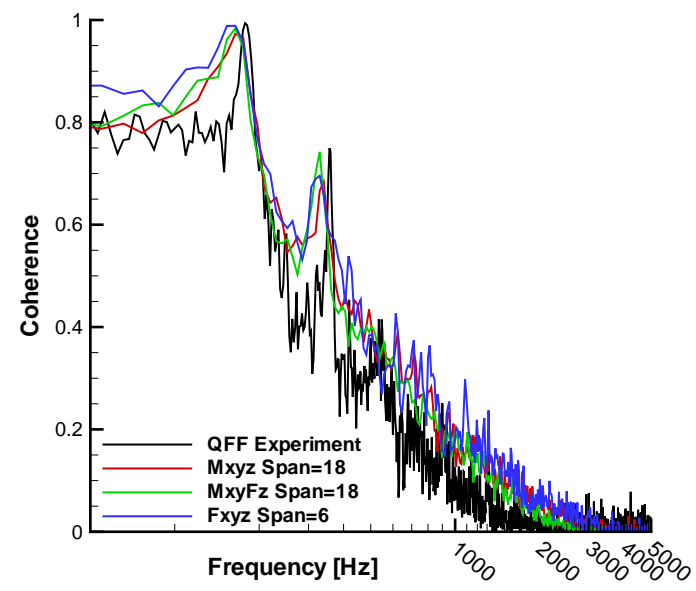

(b) Downstream cylinder

Figure 27. Coherence spectra at $90^{\circ}$ for a spanwise separation of 0.18 diameters.

\section{IV.D. Acoustic Radiation}

From a noise perspective, the downstream cylinder is going to dominate the radiation because the pressure fluctuations are 10-15 dB higher than those on the upstream cylinder. The SPL contours in the vicinity of the cylinders from figure 29(a) shows that the most intense near-field pressure fluctuations are around $\pm 45^{\circ}$ on the surface of the downstream cylinder, and in the region where the shear layers roll up behind the upstream cylinder. A more global view of the flow field in 29(b) indicates that the primary radiation direction is slightly downstream of the rear cylinder. Although the CFD seems to have directly captured some of the radiated noise, this is still very much in the near field. The wavelength of the primary shedding frequency is larger than the size of the computational domain. Despite continued advances in computational resources and numerical algorithms, it is still prohibitively expensive and often infeasible to attempt to resolve wave propagation to observer locations. Integral techniques that can predict the far-field signal based solely 


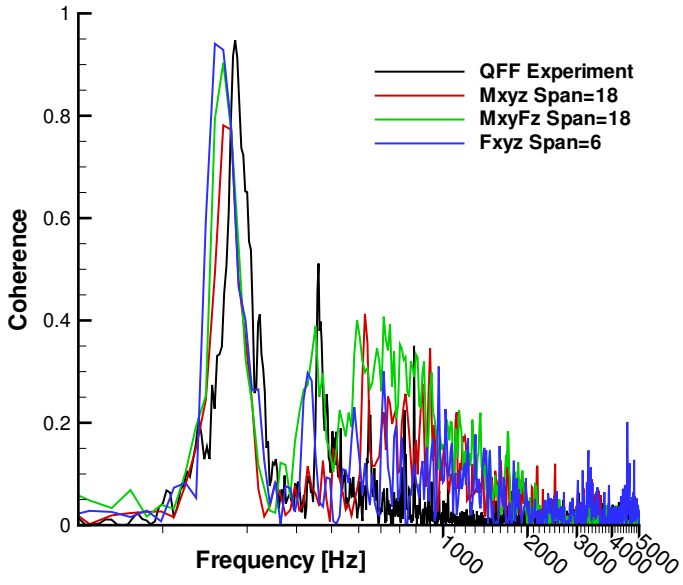

(a) Upstream cylinder

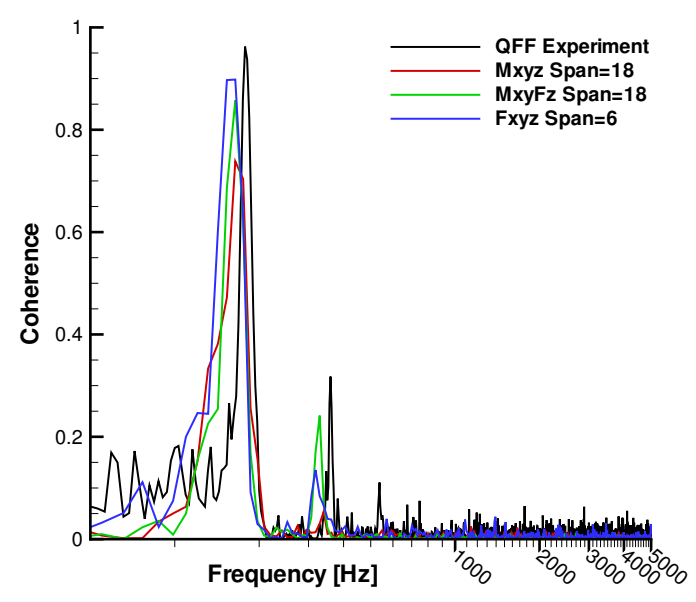

(b) Downstream cylinder

Figure 28. Coherence spectra at $90^{\circ}$ for a spanwise separation of 1 diameter.

on near-field input are a means to overcome this difficulty. Hence, the Ffowcs Williams-Hawkings (FW-H) equation ${ }^{19}$ solver described by Lockard ${ }^{20}$ is used to predict the acoustic signature at various observer locations using the unsteady flow data from the CFD calculations.

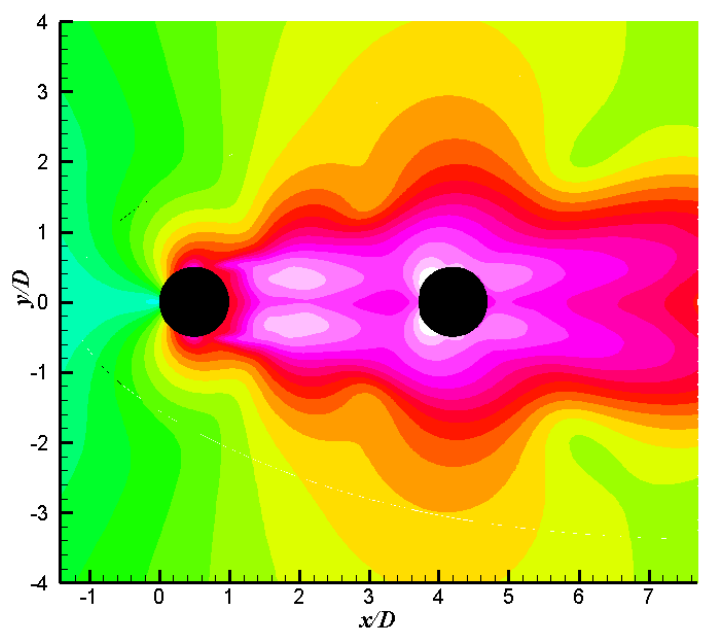

(a)

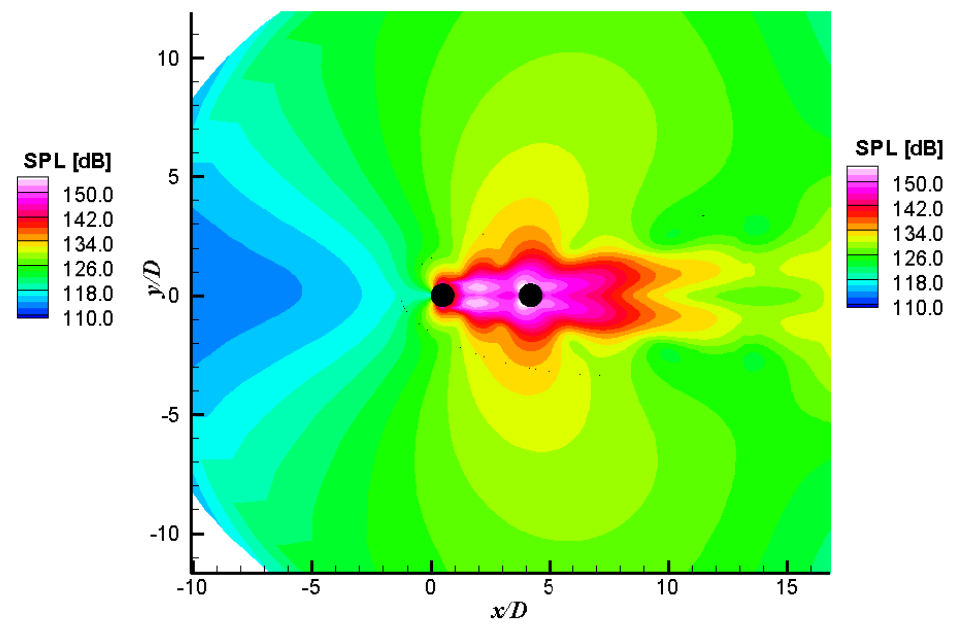

(b)

Figure 29. SPL contours from the Fxyz, span=6 calculation.

Unsteady data were collected on the surfaces shown in figure 30(a). Although the view is planar, the data were collected on full, three-dimensional surfaces that are the spanwise extensions of the lines shown in the figure. Surface 0 is comprised of the two cylinders. Surfaces 1-3 are permeable, with surfaces 1 and 2 differing only by the location of the downstream end. Surfaces 3 is farther away from the cylinders and shares part of the downstream boundary with surface 2. Directivity results for observers 1000D from the center of the upstream cylinder using the 4 surfaces is shown in figure 30(b). For the directivity plots, the pressure is nondimensionalized by $\rho_{\infty} c_{\infty}^{2}$. The calculations were performed with the frequency-domain version of the FW-H solver using 4096 samples taken over 8192 time steps. There are only minor differences between the solutions for the permeable and solid surfaces, most likely caused by vortices crossing the downstream portions of the porous surfaces. ${ }^{21,22}$ At this low frequency with a Helmholtz number $k D=0.19$, it is very unlikely that there are any flow effects on the radiated noise. Therefore, only results for the solid surfaces will be presented in the remainder of the paper.

A difficulty for the CFD that has already been mentioned is the minimal time record length available for processing. 


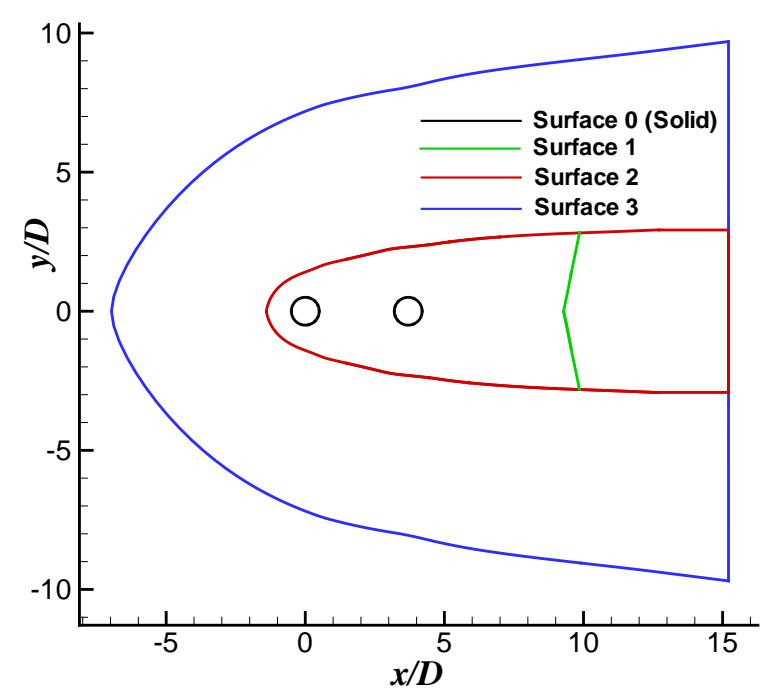

(a) FW-H Surfaces

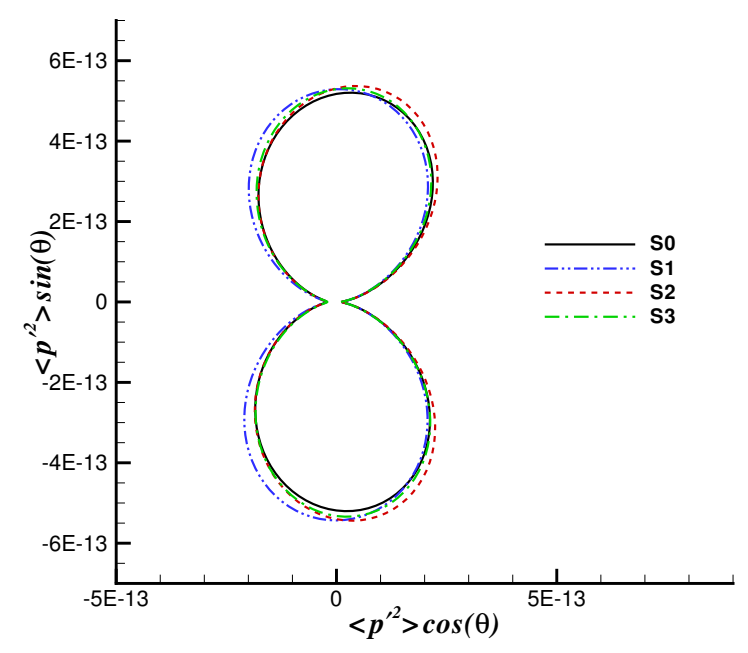

(b) MxyFz Span=18 Directivity

Figure 30. FW-H solution for observers located 1000 diameters from the center of the upstream cylinder.

For the time-averaged surfaces quantities, averaging over the span considerably improved the convergence. However, spatial averaging removes all the spanwise correlation information from the data that is needed by the FW-H solver. One could use approximate techniques ${ }^{23}$ to account for different span and correlation lengths, but these formulas are only valid in the far-field, and the experimental microphone locations do not meet this criteria. Casalino and Jacob ${ }^{13}$ have developed a statistical spanwise model that can be used with an FW-H solver to predict the noise even in the near-field. However, many problems of interest do not have a homogeneous direction, and we wished to assess whether a reasonable noise prediction can be obtained with the limited data records typical of CFD calculations. Because the CFD solutions are periodic, they can be replicated in the spanwise direction to create a cylinder of the desired length. We have chosen to use the span of 18 as the reference length for all of the noise calculations, which is very close to the QFF length of 16 diameters. However, replicating the data does not properly model the decorrelation in the spanwise direction.

To investigate the influence of the time record, we examined the results from each of 5 segments of 4096 samples from the MxyFz, span of 18 calculation. For each segment, the source terms to the FW-H equation were formed in the time domain, windowed, and then Fourier transformed. Our usual processing procedure is to average the FW-H output from all of the the segments, but in figure 31, we present the results for each segment separately along with the average. The $p^{\prime 2}$ results in 31(a) show noticeable differences which should be expected considering the variation in the time signals which have already been shown. Even greater differences between segments for other grids have been observed. However, the differences between the results for any segment and the average on a dB scale are within $2 \mathrm{~dB}$ as shown in figure 31(b). One can make noise predictions using the available data, but discrepancies of a couple of $\mathrm{dB}$ are likely because of the short time records. Using a statistical method would reduce the errors caused by the short time record, but suffer from other inaccuracies because of imperfect modeling.

Another concern with the short time records is the ability of the frequency-domain version of the FW-H to properly represent the signal. In the time-domain, an FFT of the time trace at the observer produces the desired spectra. In the frequency domain, the input to the FW-H solver must be Fourier transformed. The spectra for microphone location B from the frequency- and time-domain versions of the FW-H are compared in figure 32(a). The MxyFz span=18 solution was used for the input data. The output from 5 segments of data were averaged to produce the results. Although some slight differences can be seen, the results are very similar. Such comparisons are a good way to check that all of the data is being processed properly.

Noise predictions from the 3 grid resolutions with the longest span for each are compared with the experimental data in figures 32(b) and 33. The CFD frequency has been Strouhal scaled, and the amplitude adjusted assuming $p^{2} \sim V^{6} S t^{2}$. The levels from the Fxyz grid are the highest, presumably because the correlation never dropped much on the short span. Furthermore, the process of replicating the grid 3 times to produce a span of 18 produces an unphysical, periodic correlation. Other than overpredicting the amplitude, the agreement is fairly good. The Fxyz grid seems to do the best at picking up the peaks at the harmonics, although they are much broader than in the experimental data. Furthermore, the 3rd harmonic is equally strong for all three microphone locations, whereas it is weakest at position $\mathrm{C}$ in the experimental 


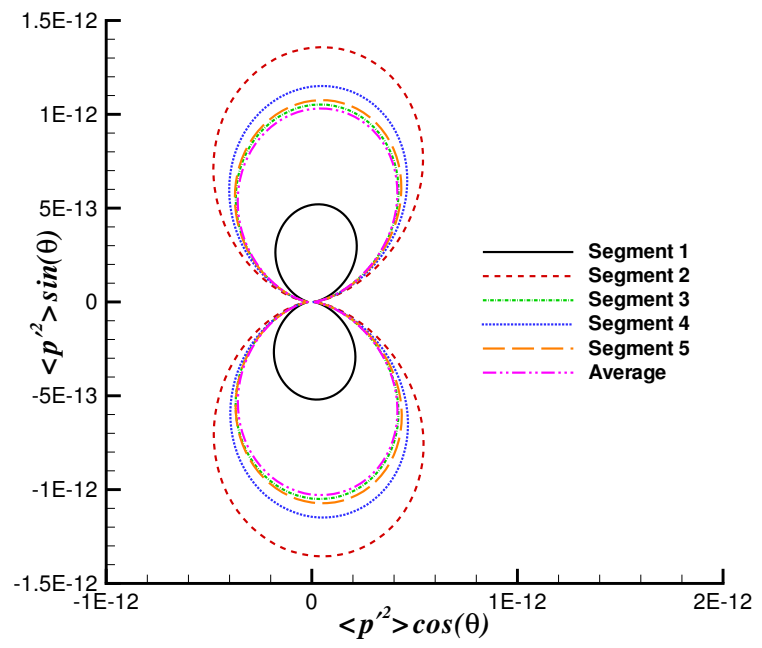

(a)

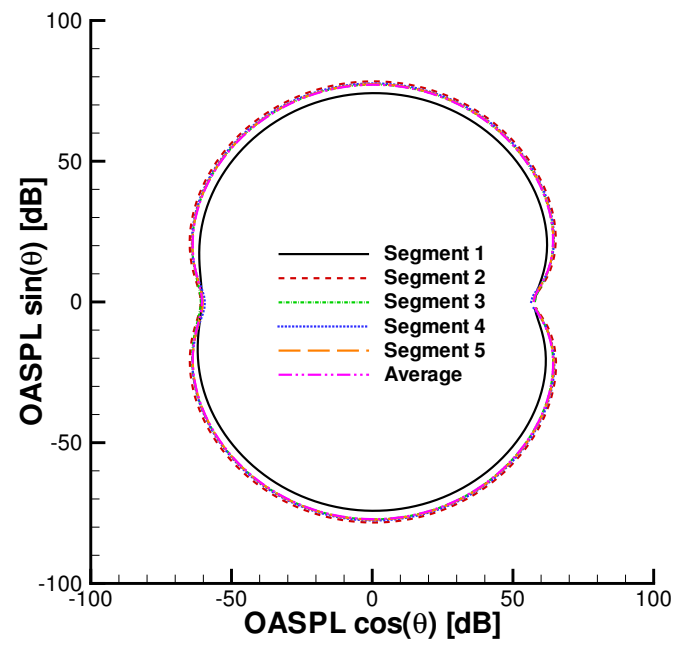

(b)

Figure 31. Directivity comparison for observers located 1000 diameters from the center of the upstream cylinder. Each segment includes 4096 samples covering 8192 time steps.

data. The noise levels from the span of 18 calculations are in good agreement with the data. The MxyFz result is slightly higher at the tone which is a reflection of the higher coherence on the finer grid as the surface fluctuations are of higher amplitude on the Mxyz grid. All of the CFD results underpredict the experiment beyond $2 \mathrm{kHz}$ which is also true of the surface pressure and most likely a grid resolution and time-step effect.

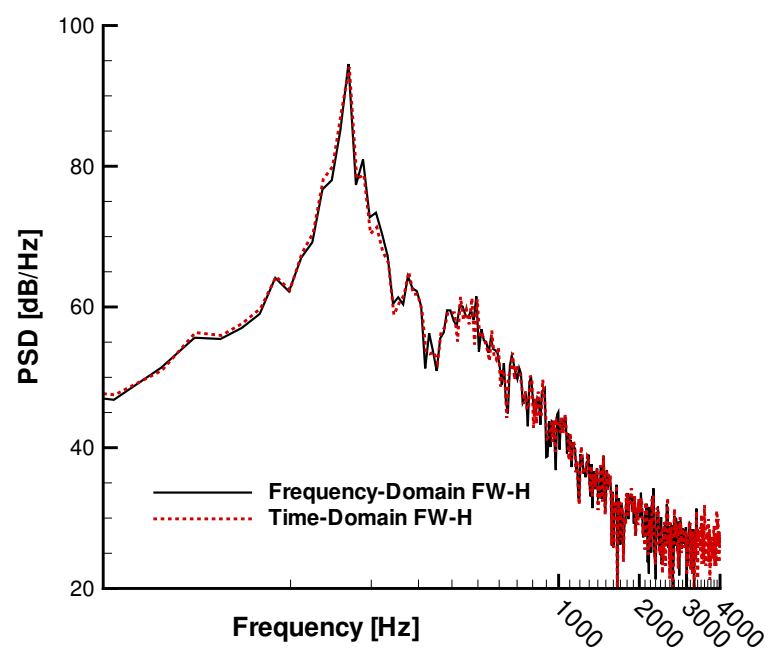

(a) $\mathrm{MxyFz}$ Span=18, point B

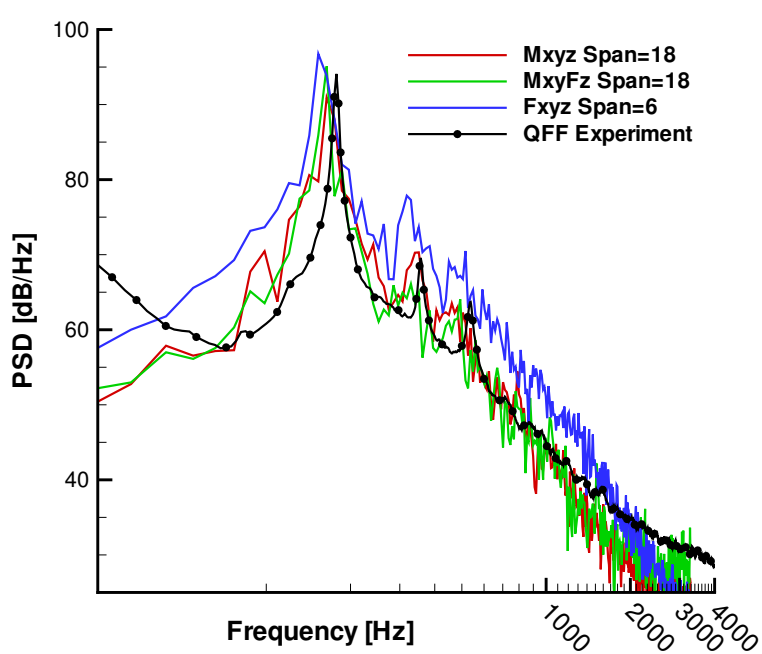

(b) Point A

Figure 32. FW-H solution for observers at points B and A.

Far-field directivities for the different numerical solutions are presented in figure 34 . The observers are located 1000 diameters from the center of the upstream cylinder. The wavelength at the tone is around 33 diameters, so this is well into the acoustic far field. The directivities peak in a slightly downstream direction and are in agreement with the near-field observations from figure 29(b). The biggest discrepancies are between the span of 18 calculations and those with shorter spans, again because of the correlation issue. The SPL plot in figure 34(b) shows that the differences are of about $5 \mathrm{~dB}$, and nonnegligible. 


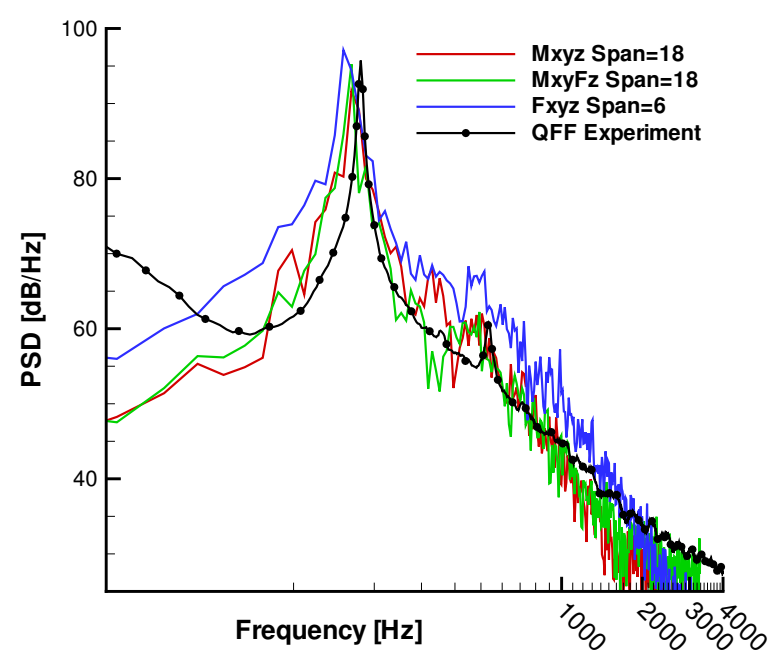

(a) Point B

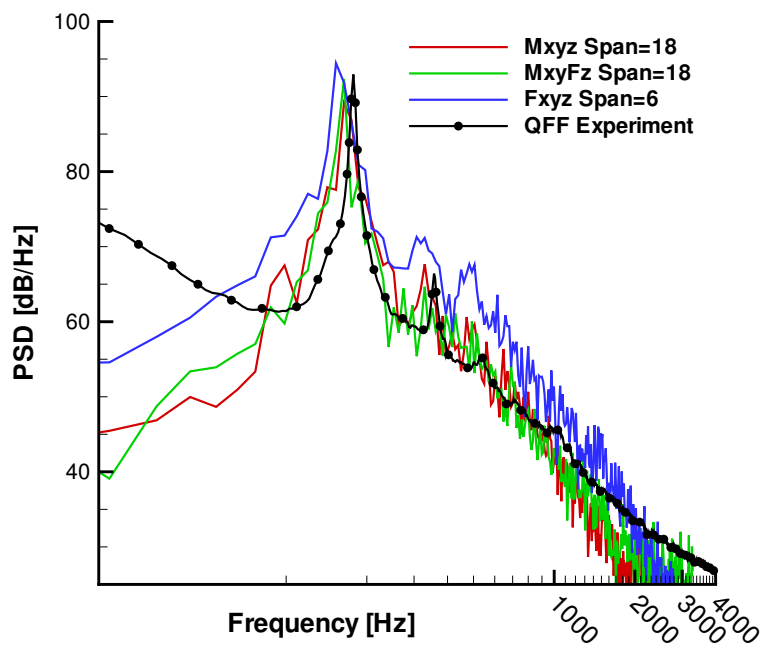

(b) Point C

Figure 33. FW-H solution for observers at points B and C.

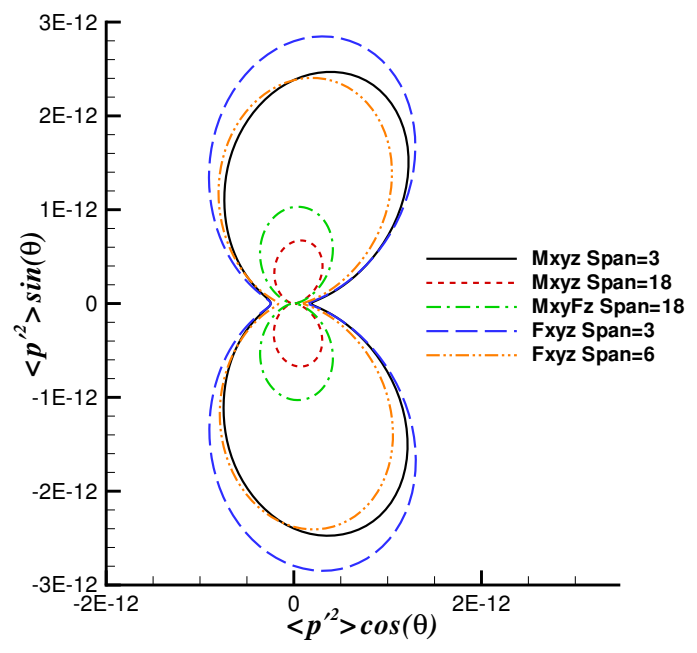

(a)

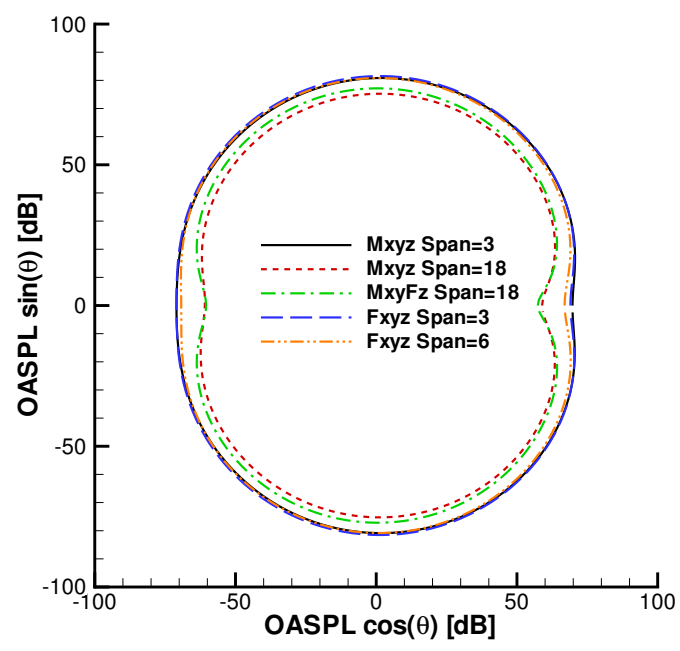

(b)

Figure 34. Directivity comparison for observers located 1000 diameters from the center of the upstream cylinder. 


\section{Conclusions}

New experimental data on inline tandem cylinder flows from the QFF have expanded the available database for code validation and demonstrated that there is only a minimal facility dependence on the results that have already been obtained in the BART. Considering that the QFF is an open-jet facility while the BART has a closed-wall test section, the relative insensitivity to the tunnel indicates that it is unnecessary to model the complete facility to obtain reasonable agreement for this particular configuration with a low blockage ratio. The QFF test has also added acoustic information, yielding a very comprehensive dataset. The current work has only presented a small portion of the data collected in the QFF. In particular, a 1/8" microphone was traversed to acquire in-flow acoustic measurements in the vicinity of the test model. Microphones on a rotating arm outside the shear layer of the open jet were used to obtain the directivity. These microphone measurements will be presented in a future paper along with a detailed description of the test.

The experiments used a trip on the upstream cylinder to insure a fully turbulent shedding process similar to that found at very high Reynolds numbers, and successfully reproduced the expected surface pressure distribution. However, the separation on the downstream cylinder is not entirely controlled, but allowed to evolve naturally in the presence of the wake of the upstream cylinder. The spanwise coherence on both cylinders was found to be quite high, which may not be fully representative of a high Re flow. Further investigation into the effects of the trip are warranted.

In general, the agreement between the experimental findings and the computations is quite good. The results underscore the need to compare CFD predictions with the full range of experimental data to get a more complete picture of the capability of CFD to simulate the overall flow. For example, even in the context of purely mean-flow statistics, surface pressures are captured quite well, but mean velocity profiles across the gap region exhibit significant discrepancies relative to the experimental values. Because of the detailed measurements of the unsteady flow, the tandem cylinder dataset should provide the necessary information to perform a thorough comparison of this kind.

Both the experiments and CFD show that the unsteady pressure fluctuation levels on the downstream cylinder are 10-15 dB higher than on the upstream cylinder. Both cylinders shed, but the interaction of the wake from the upstream cylinder is the primary cause of the high levels seen around $\pm 45^{\circ}$ on the downstream cylinder. Although these high levels are on the upstream side of the cylinder, the primary noise radiation direction is slightly downstream. The computed directivity pattern is very close to that of the classic dipole observed in isolated cylinder flows. However, the amplitude of the noise radiation is higher for the tandem cylinders.

Because of the intermittency and low frequency content in the shedding process, special emphasis was placed on determining the capability of the CFD to capture all the relevant phenomena given that very short time records are available relative to experiment. For the tandem cylinder flow under investigation, reasonable convergence in first and second moments was obtained, but the ability to average over the spanwise direction greatly aided in the convergence. Unless one resorts to a statistical method in the acoustic calculations, spanwise averaging is not possible and leads to greater variability. However, the uncertainty in OASPL caused by short time records is generally within $2-3$ decibels, which is similar in magnitude to the errors associated with methods that approximate spanwise effects. Nevertheless, one should be cognizant that short record lengths are a source of error which should be evaluated on the case-by-case basis. For problems with a homogeneous direction, the use of a statistical method will be far more efficient than directly computing the entire flow. However, information about the correlation is always needed and must be obtained either from experiment or a full computation. For the current tandem cylinder calculation, only the grids with a span of 18 were able to capture the complete decay in the correlation. Spanwise replication of the surface data from the short span simulations in the acoustic calculations overpredicted the overall radiated noise due to a lack of proper spanwise decorrelation. The overprediction was larger than the errors found when examining the effect of short temporal record lengths. Due to limitations in computational resources, we were unable to complete a computation with the finest grid and a span of 18 , which would have required 240 million grid points. We have been using a second-order CFD code due to its robustness and versatility for complex geometries, but the excessive run times required for these cylinder calculations clearly point to the need for more accuracy and efficiency to enable computations of more complex flows.

\section{Acknowledgments}

The authors would like to thank Luther Jenkins, Danny Neuhart, and Catherine McGinley for providing the BART experimental results. We would also like to acknowledge Jaye Moen and Dennis Kuchta for their help in acquiring the QFF data. 


\section{References}

${ }^{1}$ Khorrami, M. R., Lockard, D. P., Choudhari, M. M., Jenkins, L. N., Neuhart, D. H., and McGinley, C. B., "Simulations Of Bluff Body Flow Interaction For Noise Source Modeling," AIAA Paper 2006-3203 Presented at the 36th AIAA Fluid Dynamics Conference and Exhibit, San Francisco, CA, United States, June 5-8, 2006.

${ }^{2}$ Jenkins, L. N., Khorrami, M. R., Choudhari, M. M., and McGinley, C. B., "Characterization of Unsteady Flow Structures Around Tandem Cylinders for Component Interaction Studies in Airframe Noise," AIAA Paper 2005-2812 Presented at the 11th AIAA/CEAS Aeroacoustics Conference, Monterey, CA, May 23-25, 2005.

${ }^{3}$ Jenkins, L. N., Neuhart, D. H., McGinley, C. B., Choudhari, M. M., and Khorrami, M. R., "Measurements of Unsteady Wake Interference Between Tandem Cylinders," AIAA Paper 2006-3202 Presented at the 36th AIAA Fluid Dynamics Conference and Exhibit, San Francisco, CA, United States, June 5-8, 2006.

${ }^{4}$ Hutcheson, F. V. and Brooks, T. F., "Noise Radiation from Single and Multiple Rod Configurations," AIAA Paper 2006-2629 Presented at the 12th AIAA/CEAS Aeroacoustics Conference, Cambridge, MA, May 8-10, 2006, 2006.

${ }^{5}$ Khorrami, M. R., Choudhari, M. M., Jenkins, L. N., and McGinley, C. B., "Unsteady Flowfield Around Tandem Cylinders as Prototype for Component Interaction in Airframe Noise," AIAA Paper 2005-2866 Presented at the 11th AIAA/CEAS Aeroacoustics Conference, Monterey, CA, May 23-25, 2005.

${ }^{6}$ Khorrami, M. R., Choudhari, M. M., Lockard, D. P., Jenkins, L. N., and McGinley, C. B., "Unsteady Flowfield Around Tandem Cylinders as Prototype for Component Interaction in Airframe Noise," Accepted by AIAA Journal. 1605.

${ }^{7}$ Menter, F. R., "Two-equation Eddy-viscosity Turbulence Models for Engineering Applications," AIAA Journal, Vol. 32, No. 8, 1994, pp. 1598-

${ }^{8}$ Zdravkovich, M. M., "Review of Flow Interference Between Two Circular Cylinders in Various Arrangements," Journal of Fluids Engineering, Vol. 99, 1977, pp. 618-633.

${ }^{9}$ Zdravkovich, M. M., Flow Around Circular Cylinders Volume 1, Oxford University Press, 1997.

${ }^{10}$ Zdravkovich, M. M., Flow Around Circular Cylinders Volume 2: Applications, Oxford University Press, 2002.

${ }^{11}$ Zdravkovich, M. M., "Flow Induced Oscillations of Two Interfering Circular Cylinders," Journal of Sound and Vibration, Vol. 4, 1985, pp. 511521

${ }^{12}$ Roshko, A., "Experiments On The Flow Past A Circular Cylinder At Very High Reynolds Number," Journal of Fluid Mechanics, Vol. 10, 1961, pp. $345-356$.

${ }^{13}$ Casalino, D. and Jacob, M., "Prediction of Aerodynamic Sound from Circular Rods via Spanwise Statistical Modeling," Journal of Sound and Vibration, Vol. 262, No. 4, 2003, pp. 815-844.

${ }^{14}$ Fujita, H., Suzuki, H., Sagawa, A., and Takehaisa, T., "The Aeolian Tone and the Surface Pressure in High Reynolds Number Flow," AIAA Paper 2000-2002, Presented at the 21st AIAA Aeroacoustics Conference, Lahaini, HI, 2000.

${ }^{15}$ Szepessy, S., "On the Spanwise Correlation of Vortex Shedding from a Circular Cylinder at High Subcritical Reynolds Number," Physics of Fluids, Vol. 6, No. 7, 1994, pp. 2406-2416.

${ }^{16}$ Rumsey, C. L., Biedron, R. T., and Thomas, J. L., "CFL3D: Its History and Some Recent Applications," NASA TM 112861, May 1997, presented at the Godonov's Method for Gas Dynamics Symposium, Ann Arbor, MI.

${ }^{17}$ Krist, S. L., Biedron, R. T., and Rumsey, C. L., “CFL3D User's Manual (Version 5),” NASA TM 208444, NASA Langley Research Center: Computational Aerosciences Branch, 1997.

${ }^{18}$ Menter, F. R., "Zonal Two-equation k-w Turbulence Models for Aerodynamic Flows,” AIAA Paper 1993-2906, 1993.

${ }^{19}$ Ffowcs Williams, J. E. and Hawkings, D. L., "Sound generation by turbulence and surfaces in arbitrary motion," Philosophical Transactions of the Royal Society of London A, Vol. 342, 1969, pp. 264-321.

${ }^{20}$ Lockard, D. P., "A Comparison of Ffowcs Williams-Hawkings Solvers for Airframe Noise Applications," AIAA Paper 2002-2580, 8th AIAA/CEAS Aeroacoustics Conference, Breckenridge, CO, June 17-19, 2002.

${ }^{21}$ Lockard, D. P. and Casper, J. H., "Permeable Surface Corrections for Ffowcs Williams and Hawkings Integrals," AIAA Paper 2005-2995, Presented at the 11th AIAA/CEAS Aeroacoustics Conference, Monterey, CA, 2005.

${ }^{22}$ Cheung, L. C. and Lele, S. K., "Evaluation of Far-Field Sound with Open Surface Corrections to Lighthill's Equation," AIAA Paper 2006-2488, Presented at the 12th AIAA/CEAS Aeroacoustics Conference, Cambridge, MA, 2006.

${ }^{23}$ Seo, J. H., Chang, K. W., and Moon, Y. J., "Aerodynamic Noise Prediction for Long-Span Bodies," AIAA Paper 2005-2573, Presented at the 12th AIAA/CEAS Aeroacoustics Conference, Cambridge, MA, May 8-10, 2006. 